Published in final edited form as:

Nat Neurosci. 2018 December ; 21(12): 1728-1741. doi:10.1038/s41593-018-0262-6.

\title{
Electrical spinal cord stimulation must preserve proprioception to enable locomotion in humans with spinal cord injury
}

\author{
E. Formento ${ }^{1,2}$, K. Minassian², F. Wagner², JB. Mignardot ${ }^{2}$, C. G. Le Goff ${ }^{2}$, A. Rowald ${ }^{2,3}$, J. \\ Bloch $^{4}$, S. Micera ${ }^{1,5,6}$, M. Capogrosso ${ }^{3,6}$, and G. Courtine ${ }^{2,4,6, \dagger}$
}

${ }^{1}$ Bertarelli Foundation Chair in Translational NeuroEngineering, Institute of Bioengineering, Swiss Federal Institute of Technology (EPFL), Lausanne, Switzerland ${ }^{2}$ Center for Neuroprosthetics and Brain Mind Institute, School of Life Sciences, Swiss Federal Institute of Technology (EPFL), Lausanne, Switzerland ${ }^{3}$ Department of Medicine, Faculty of Sciences, University of Fribourg. Fribourg, Switzerland ${ }^{4}$ Department of Neurosurgery, University Hospital of Lausanne (CHUV), Lausanne, Switzerland ${ }^{5}$ Neural Engineering Area, Institute of Biorobotics, Scuola Superiore Sant'Anna, Pisa, Italy

\section{Abstract}

Epidural electrical stimulation (EES) of the spinal cord restores locomotion in animal models of spinal cord injury (SCI) but is less effective in humans. Here, we hypothesized that this interspecies discrepancy is due to interference between EES and proprioceptive information in humans. Computational simulations, preclinical and clinical experiments reveal that EES blocks a significant amount of proprioceptive input in humans, but not in rats. This transient deafferentation prevents the modulation of reciprocal inhibitory networks involved in locomotion and reduces or abolishes the conscious perception of leg position. Consequently, continuous EES can only facilitate locomotion within a narrow range of stimulation parameters and is unable to provide meaningful locomotor improvements in humans without rehabilitation. Simulations showed that burst stimulation and spatiotemporal stimulation profiles mitigate the cancellation of proprioceptive information, enabling robust control over motoneuron activity. This demonstrates

Users may view, print, copy, and download text and data-mine the content in such documents, for the purposes of academic research, subject always to the full Conditions of use:http://www.nature.com/authors/editorial_policies/license.html\#terms

†To whom correspondence should be addressed: Grégoire Courtine (gregoire.courtine@epfl.ch).

${ }^{6}$ Co-senior author

Data availability

Acquired data are available from the corresponding author upon reasonable request.

Code availability

Software routines developed for data analysis are available from the corresponding author upon reasonable request. The code to perform and analyze the neural simulations is available as supplementary information and at https://github.com/FormentoEmanuele/ MuscleSpindleCircuitsModel.git.

Authors contributions

E.F., M.C., K.M., S.M., and G.C. conceived the study. E.F. and M.C. designed the computational model and E.F. performed the simulations. J.B. performed the surgery in humans. E.F., K.M., F.W., JB.M. and C.G.LG. performed the experiments. A.R. and E.F. built the robotic platform to control rat ankle kinematics. E.F. performed the data analyses and prepared the figures. G.C. wrote the manuscript with E.F., M.C., K.M, and all the authors contributed to its editing. G.C., S.M., M.C. and J.B. supervised the work.

Competing interests

G.C. and S.M. are founders and shareholders of GTXmedical SA, a company developing neuroprosthetic systems in direct relationship with the present work. E.F., M.C., G.C. and S.M. hold several patents related to electrical spinal cord stimulation. 
the importance of stimulation protocols that preserve proprioceptive information to facilitate walking with EES.

\section{Introduction}

Spinal cord injury (SCI) has an immediate and devastating impact on movement control. These motor deficits result from the interruption of communication between the brain and spinal cord, depriving the otherwise intact spinal cord executive centers below the injury from essential sources of modulation and excitation to produce movement1.

Epidural electrical stimulation (EES) applied to the lumbar spinal cord immediately enables the executive centers to coordinate a broad range of motor behaviors including standing, walking in various directions, and even running in rodent, feline, and nonhuman primate models of leg paralysis2-5. When combined with locomotor training, EES promotes an extensive reorganization of residual neural pathways that restored locomotion without the need of stimulation2,6.

EES has also been applied to the human spinal cord for several decades but has been less effective. EES induced rhythmic leg movements in people with complete paralysis7,8, and enabled independent stepping when delivered over more than a year of intense rehabilitation9-11. EES also enabled volitional activation of paralyzed muscles to initiate isolated leg movements in individuals with motor complete paralysis12,13. However, EES has not restored independent, weight-bearing locomotion in humans with severe SCI, as observed in animal models.

The mechanisms underlying species-specific responses to EES remain enigmatic. This understanding is essential for guiding the development of evidence-based approaches that fulfill the potential of EES to improve recovery after SCI.

Evidence from computational models14,15 and experimental studies $16-18$ conducted in animals and humans suggests that EES recruits afferent fibers conveying proprioceptive information. This recruitment leads to the activation of motoneurons through monosynaptic and polysynaptic proprioceptive circuits, and increases the overall excitability of the lumbar spinal cord. This modulation enhances the responsiveness of spinal circuits to residual descending signals and sensory feedback. In turn, sensory information modulates the reciprocal inhibitory networks in the spinal cord that gate the excitatory drive produced by EES towards functionally relevant pathways. This mechanism enables the generation of muscle activation underlying standing and walking in animal models of paralysis 18 .

This conceptual framework implies that sensory information plays a central role in motor pattern formation during EES. However, this viewpoint does not consider that the recruitment of proprioceptive fibers by EES may interfere with the natural flow of information traveling along the same fibers.

Electrical stimulation triggers bi-directional action potentials (APs) along the recruited fiber. EES would thus elicit orthodromic and antidromic APs that travel to the spinal cord and 
sensory organs 19-21. Consequently, we hypothesized that antidromic APs may collide with APs conveying proprioceptive information, preventing its propagation to the brain and spinal cord. The probability of these detrimental interactions is proportional to EES frequency, the firing rate of afferents, and the time required for an AP to travel along the entire length of the fiber. These physiological parameters diverge dramatically between rats and humans. The traveling time of APs along proprioceptive fibers is longer in humans compared to rats, and firing rates are lower22. The resulting higher probability of collisions between natural and antidromic APs in humans may disrupt sensory information. Here, we hypothesized that this phenomenon explains the limited efficacy of continuous EES in paraplegic individuals compared to rats.

We demonstrate that antidromic collisions abolish proprioceptive information in humans, but not in rats. These detrimental interactions restrict the range of EES frequencies and amplitudes that can facilitate locomotion. We report EES strategies that mitigate this issue, demonstrating that EES must preserve proprioception to facilitate walking after SCI.

\section{Results}

\section{Antidromic collisions during EES}

To study the occurrence probability of antidromic collisions along proprioceptive afferents during EES, we developed computational models of proprioceptive afferents that consider the length of axons innervating proximal and distal muscles, and the propagation times of APs. We modeled realistic interactions between natural and EES-elicited APs (Figure 1a). We thus calculated the probability of antidromic collisions in muscle spindle afferents depending on EES frequency and natural firing rate.

The occurrence probability of antidromic collisions was extremely low in rats, regardless of EES frequency and natural firing rate (Figure 1b). While delivering EES at frequencies commonly used to enable locomotion in rats $(40 \mathrm{~Hz} 2,23)$, this probability never exceeded $20 \%$.

These probabilities were dramatically different in humans. Even relatively low EES frequencies blocked most of the natural proprioceptive signals from reaching the spinal cord. For distal muscles, the occurrence probability of antidromic collisions reached nearly $100 \%$ for afferent firing rates of 30 impulses per second (Imp/s) at 30Hz EES frequency (Figure 1c). The occurrence probability of antidromic collisions was markedly higher along afferents innervating proprioceptors located in distal muscles compared to proximal muscles (Figure 1c).

These results suggest that continuous EES may disrupt proprioceptive information in humans, but not in rats.

\section{EES induces antidromic activity along human afferents}

We thus verified whether EES produces antidromic activity along proprioceptive afferents. We recorded the proximal and distal branches of the tibial nerve (mixed nerve), the sural 
nerve (sensory nerve), and EMG activity from the soleus muscle during continuous EES in two individuals with chronic SCI (Figure 2a; Subject \#2 and \#3 in Supplementary Table 1).

We selected an EES configuration that elicited contractions of the soleus and then reduced EES amplitude to elicit a tingling sensation in the corresponding dermatome without visible muscle contraction. In subject \#2, each pulse of EES $(20 \mathrm{~Hz})$ elicited a weak response in the soleus with a latency of $25 \mathrm{~ms}$ that has been associated with the recruitment of motoneurons via group-Ia afferents15. Concurrently, we detected two responses in the proximal branch of the tibial nerve, with latencies of 12.5 and $26.5 \mathrm{~ms}$, and one response (latency, $21 \mathrm{~ms}$ ) in the distal branch. The responses induced in the proximal $(12.5 \mathrm{~ms})$ and distal $(21 \mathrm{~ms})$ branches of the tibial nerve (Figure 2a, blue windows) likely resulted from the same neural volley propagating towards the periphery. Since the responses recorded in the distal branch occurred prior to any motor response, they cannot be attributed to orthodromic efferent activity. These responses corresponded to antidromic afferent volleys. The response (22 ms) recorded in the exclusively sensory sural nerve is compatible with this conclusion. The antidromic recruitment of $A \beta$ afferents is the most probable explanation for this response. In subject \#3, each EES pulse elicited a distinct response in both proximal $(12.5 \mathrm{~ms})$ and distal ( $22 \mathrm{~ms}$ ) branches of the tibial nerve, and a response in the sural nerve $(22.5 \mathrm{~ms})$. No responses were detected in the soleus muscle.

These results indicate that EES elicits antidromic activity along proprioceptive afferents, suggesting that EES interferes with natural sensory information in humans.

\section{EES disrupts kinesthesia}

Cancellation of proprioceptive information during EES should alter the conscious perception of joint position and movement velocity. To test this hypothesis, three individuals with a chronic SCI (Supplementary Table 1) completed a threshold to detection of passive movement (TTDPM) test. Due to impaired sensory function, only subject \#1 and subject \#3 could complete the task without EES (Figure 2b).

Participants sat in a robotic system that imposed a passive isokinetic leg movement (Figure 2c). They were asked to detect the direction of movement as soon as they could perceive it, but before the knee joint angle reached a predefined amplitude.

Without EES, subject \#1 detected extension and flexion of the knee with $100 \%$ success (median detection angle: $7 \mathrm{deg}$, 95\% CI: 3.9-11.9 deg). Without stimulation, subject \#3 successfully detected movement onset with $100 \%$ success (median detection angle: $6.7 \mathrm{deg}$, 95\% CI: 5.8-8.4 deg).

We selected electrode configurations that targeted antagonistic muscles of the knee. We first tested amplitudes that elicited a tingling sensation without producing motor responses (x 0.8 muscle response threshold). At this intensity and over a broad range of frequencies, continuous EES did not alter subject \#1's performance, while detection of movement onset was disrupted in subject \#3 (Figure $2 \mathrm{~d}$ and Supplementary Figure 1). At 1.5x muscle response threshold, EES prevented both participants from detecting leg movements. The participants reported a complete loss of awareness of leg position and movement. 
These psychophysical experiments corroborate our hypothesis that continuous EES disrupts and may even block proprioceptive information in humans. This disruption occurred at amplitudes and frequencies commonly used for rehabilitation8,12,13.

\section{Continuous EES alters afferent modulation of spinal circuits in humans but not in rats}

Proprioceptive signals exert a strong influence on the excitability of sensorimotor circuits 24-26. The cancellation of proprioceptive information during continuous EES in humans should therefore affect the modulation of reflex responses elicited by EES.

To test this hypothesis, we studied the modulation of reflex responses elicited by various EES frequencies ( 5 to $60 \mathrm{~Hz}$ ) during passive oscillations of the ankle or knee joint. The participants were seated in a robotic system that imposed passive rhythmic flexion-extension movements of the ankle or knee at a fixed angular velocity and amplitude (Figure 3a and Supplementary Figure 2). Continuous EES was delivered with electrode configurations and intensities that induced reflex responses in flexor and extensor muscles of the targeted joint (Figure 3a and Supplementary Figure 2).

The rhythmic flexion-extension movements of the joint induced a significant phasedependent modulation of reflex responses in the mobilized muscles (normalized modulation depth superior to $0.3 ; \mathrm{p}<0.05$ for each frequency, bootstrap; Figure 3b-d). However, the extent of this modulation depth depended on EES frequency. Quantification of angledependent reflex responses revealed a pronounced monotonic decrease of the normalized modulation depth with EES frequency increments (Figure 3d).

We performed the same experiments in four lightly anesthetized rats with a contusion SCI that had been implanted with an electrode over the lumbar spinal cord (Figure 3e-h). Robotcontrolled oscillations of the ankle induced a robust modulation of reflex responses (normalized modulation depth superior to $0.18 ; \mathrm{p}<0.05$ for each frequency, bootstrap). However, we did not detect systematic relationships between EES frequencies and normalized modulation depth (Figure 3h). Modulation of motor responses was still present at frequencies as high as $100 \mathrm{~Hz}$ (Figure 3g). A linear fit of the median values yielded a slope close to 0 in all rats (median $=0.0003 ; 95 \%$ confidence interval $=[-0.0056,0.0015]$, bootstrap), suggesting a lack of linear dependency between modulation depth and EES frequency.

These experiments indicate that continuous EES disrupts the ability of proprioceptive information to modulate the motor output elicited by EES.

\section{Computational models of proprioceptive feedback circuits during locomotion}

We next sought to assess the impact of continuous EES on the natural dynamics of proprioceptive feedback circuits during locomotion. Since these interactions cannot be studied in vivo, we synthesized EES properties, proprioceptive feedback circuits, and leg biomechanics into computational models (Figure 4a). We adapted a previously validated dynamic computational model18 to the anatomical features of rats and humans. The model includes the minimal proprioceptive neural network responsible for reciprocal activation of antagonist muscles (Figure 4b). We used species-specific biomechanical and muscle-spindle 
models to estimate the firing rates of proprioceptive afferents during locomotion. This afferent activity was used to steer the neural networks (Figure 4c).

We first studied the impact of EES on the activity of proprioceptive afferents. To model increments in EES amplitude and frequency, we scaled up the number of recruited afferent fibers and the rates of both orthodromic and antidromic induced activities, respectively. In rats, EES did not alter the modulation depth of proprioceptive information (Figure 4d). In striking contrast, the same parameters of EES dramatically disrupted the modulation of proprioceptive information in humans. With frequencies as low as $40 \mathrm{~Hz}$, antidromic action potentials abolished the sensory information conveyed by each electrically stimulated fiber. The residual modulation of proprioceptive information resulted solely from the activity of non-recruited afferent fibers. The percentage of erased proprioceptive information was directly proportional to EES amplitude (Figure 4d).

We then evaluated the impact of this cancellation on the ability of EES to steer reciprocal activation of motoneurons innervating antagonist muscles during locomotion. Continuous EES delivered excitation to Ia-inhibitory interneurons and motoneurons. In rats, the modulation of Ia-inhibitory interneurons driven by the natural proprioceptive information led to a reciprocal activation of antagonist motoneurons during the stance and swing phases of gait (Figure 5a). Increasing EES frequency or amplitude resulted in higher firing rates of motoneurons, but only during their natural phase of activity.

In the human model, antidromic collisions dramatically disrupted the dynamics of the neural network (Figure 5b). At low frequency and low amplitude, continuous EES steered the reciprocal activation of antagonist motoneurons, as observed in rats. With higher stimulation parameters, the cancellation of proprioceptive information prevented phase-dependent modulation of Ia-inhibitory interneurons. The resulting imbalance between antagonist pools of Ia-inhibitory interneurons led to a profound asymmetry in the excitatory drive delivered to motoneurons. Extensor motoneuron pools became over-active while flexor motoneuron pools received strong inhibition (Figure 5b).

These results suggest that only a narrow range of EES parameters could be exploited to enhance the excitability of the human spinal cord without compromising the critical role of proprioceptive information in the production of locomotion. Therefore, the degree of controllability over human motoneurons may be very limited compared to rats.

\section{Limited facilitation of locomotion in humans compared to rats}

We then evaluated the impact of EES frequencies and amplitudes on leg muscle activity during locomotion in rats and humans.

Rats with a clinically-relevant contusion SCI6 and EES electrodes $(n=4$ rats) were positioned bipedally in a bodyweight support system over a treadmill (Figure 6a). Continuous EES $(40 \mathrm{~Hz})$ induced robust locomotor movements of the otherwise paralyzed legs (Figure 6b). As previously reported3,18,27, increases in EES frequencies (20-80 Hz) led to a linear modulation of leg muscle activity, which gradually adjusted kinematic features such as step height (Figure 6b,c). 
The three participants with SCI were supported by a gravity-assist 28 that provided trunk support to facilitate stepping on a treadmill (Figure 6d). Using rails located on each side of the treadmill, subject \#1 (60\% body weight support) and subject \#2 (70\% body weight support) were able to take some steps on the moving treadmill belt and produce alternating activation of antagonist leg muscles without EES. However, this muscle activity did not translate into functional movements, as both feet dragged along the treadmill belt at the end of stance. The amplitude of leg movements remained limited. Continuous EES ( $40 \mathrm{~Hz}, 3$ to $9 \mathrm{~mA}$ ) facilitated leg muscle activity and kinematic features (Figure 6e,f and Supplementary Figures 3 and 4). Contrary to rats, however, this facilitation was insufficient to enable coordinated, weight-bearing locomotion. Subject \#3 exhibited flaccid paralysis of all leg muscles. Continuous EES increased muscle activity, but failed to produce consistent modulation of this activity to produce stepping (Supplementary Figure 5). All participants reported a complete loss of limb position awareness during continuous EES, which affected their ability to coordinate the timing of locomotor movements.

Consequently, we sought to augment muscle activity with increases in EES frequency or amplitude. From optimal EES parameters, increases in frequency or amplitude did not improve stepping. The amplitude of EMG activity scaled up in flexor muscles, but this increase was associated with a concomitant decrease in extensor muscles, even leading to a complete suppression of extensor muscle activity (Figure 6e,f and Supplementary Figures 3 and 4). EES often induced co-activation of antagonist muscles, with the occurrence of abnormal bursting activity in flexor muscles during stance. Co-activation of muscles induced a sensation of stiff legs, reflected in the reduced range of motion of leg joints (Figure 6e,f and Supplementary Figures 3 and 4).

These results are consistent with our simulations, indicating that the range of useful EES parameters are too narrow to enable robust locomotion in humans without training, thus providing a plausible explanation for inter-species differences in the therapeutic impact of continuous EES.

\section{Spatiotemporal EES protocols may remedy the limitations of continuous EES}

We next exploited our computational model to identify stimulation strategies that may remedy the identified limitations of continuous EES.

We reasoned that, to avoid disrupting the natural network dynamics, the temporal and spatial structure of EES should encode the profile of proprioceptive feedback information. We surmised that the amplitude / frequency of the stimulation targeting a specific muscle should be proportional to the instantaneous firing rate of the proprioceptive afferents originating from the sensory organs located in this muscle. Due to the continuous match between the proprioceptive afferent activity and the stimulation profile, EES would augment the overall excitation delivered to the targeted motor pool without compromising the information conveyed by the proprioceptive afferents. Targeting antagonist motor pools with their specific stimulation profile would contribute to maintaining the modulation of reciprocal inhibitory networks that is necessary to facilitate walking with EES. In turn, we hypothesized that adjusting the amplitude and frequency used to configure the stimulation profiles would enable controlling the activity of motoneurons. 
We implemented this stimulation strategy in the computational model. We constructed stimulation profiles that combined the natural modulation of primary and secondary proprioceptive afferents (group-Ia, group-II, and Ib, Figure 7a,b) from the homonymous muscles. We did not explicitly model Golgi tendon organs, although Ib-afferents are also recruited with EES and provide strong excitation during locomotion29. Because of the close correlations between Ib-afferent firings and homonymous muscle activity30, the EMG envelope was used as a surrogate for the firing profile of Ib-afferents.

Simulations revealed that this strategy erased proprioceptive information to a similar extent as continuous EES (Figure 7c). Due to the continuous match between the natural proprioception and stimulation profile, however, the proprioceptive signals reaching the spinal cord contained the same amount of information. Naturally-generated APs annihilated by antidromic collision were replaced by EES-produced orthodromic APs. While the percentage of erased information increased with EES amplitude (Figure 7c), the depth of proprioceptive afferent modulation remained preserved, or even increased for higher stimulation amplitudes. Consequently, the stimulation artificially drove the reciprocal modulation of Ia-inhibitory interneurons, as would the natural proprioception during walking (Figure 7c). Scaling up EES amplitude led to a proportional increase in the firing rates of proprioceptive afferents, which augmented the excitation delivered to motoneurons. Since this excitation was restricted to the active phase of each motoneuron pool, increasing EES parameters enabled a linear modulation of motoneuron firing rates (Figure 7c).

These results suggest that encoding the profile of proprioceptive afferent activity into the spatiotemporal structure of EES protocols may expand and refine the control over the amplitude of motoneuron activity while also reinforcing the modulation of reciprocal inhibitory networks, thereby enhancing the facilitation of walking compared to continuous EES.

\section{High-frequency low-amplitude EES alleviates the disruptive effects of continuous EES}

We finally explored whether alternative strategies based on continuous EES could alleviate the cancellation of proprioception.

We sought to design a stimulation strategy that minimizes the amount of erased proprioceptive information during continuous EES while providing high post-synaptic excitation to motoneurons. Each Ia-afferent synapses onto every motoneuron that innervates the homonymous muscle31,32. Moreover, high-frequency stimulation of nerve afferents leads to a temporal summation of excitatory post-synaptic potentials (EPSP) delivered to the targeted cell33-35. We concluded that recruiting a limited number of Ia-afferents with a stimulation burst of low amplitude but high frequency could theoretically deliver the same excitation to motoneurons as the recruitment of a large number of Ia-afferents with single pulses of high amplitude. We thus hypothesized that each pulse of EES could be replaced by a high-frequency, low-amplitude burst of EES that would provide the same overall excitation to motoneurons while reducing the overall amount of erased proprioceptive information. Indeed, while the proprioceptive information traveling along the recruited fibers would still be blocked by the stimulation, the reduced number of electrically recruited afferents would ensure that a large amount of fibers remain able to convey sensory signals to the spinal cord. 
Finally, the excitation delivered to motor pools could then be controlled by adjusting the inter-burst interval.

We tested the hypotheses underlying this stimulation strategy using computer simulations with multicompartmental motoneuron models and realistic distribution of Ia-afferent synaptic contacts (Figure 8a). As predicted, the temporal summation of EPSPs elicited by high-frequency low-amplitude bursts of stimulation enabled recruiting the same number of motoneurons as single pulses of high amplitude EES (Figure 8b).

To validate these results experimentally, we conducted electrophysiological experiments in five rats. Figure $8 \mathrm{c}$ shows motor responses recorded in the tibialis anterior during single pulses and single bursts of EES ( $25 \mathrm{~ms}$ duration, frequencies: 100 to $1000 \mathrm{~Hz}$ ) at increasing amplitudes. Compared to single pulses, high-frequency burst stimulation decreased the threshold to elicit a motor response by $39.8 \%$ (SEM: $\pm 4.4 \%$ ). The largest reductions were obtained towards $500 \mathrm{~Hz}$ (SEM: $\pm 54.8 \mathrm{~Hz}$ ). Decreases in EES burst amplitude led to increased latencies of motor responses, suggesting that a higher number of pulses was necessary to recruit motoneurons through the temporal summation of EPSPs (Figure 8d).

The pulse generator implanted in the participants could generate waveforms with a maximum frequency of $125 \mathrm{~Hz}$. However, the simultaneous delivery of interleaved waveforms ( $2 \mathrm{~ms}$ hard-coded delay) enabled the configuration of single bursts composed of 4 pulses delivered at $500 \mathrm{~Hz}$. This feature allowed us to evaluate the concept of highfrequency EES in humans. As observed in rats, high-frequency bursts of EES required markedly reduced stimulation amplitudes to elicit a motor response compared to single pulses (Figure 8e,f).

We implemented this stimulation strategy into the computational model. We delivered EES bursts consisting of 5 pulses at $600 \mathrm{~Hz}$ with a stimulation amplitude recruiting 20\% of all primary afferents. Compared to continuous EES, this stimulation reduced the amount of erased proprioceptive information (Supplementary Figure 6). Decreasing the time between each EES burst led to a proportional increase in the excitation delivered to motoneurons.

These results suggest that high-frequency, low-amplitude stimulation protocols may alleviate the detrimental impact of continuous EES on the modulation of proprioceptive feedback circuits in humans.

\section{Discussion}

We have accumulated evidence that the antidromic recruitment of proprioceptive afferents during continuous EES blocks the propagation of naturally-generated proprioceptive signals to the brain and spinal cord. Computer simulations suggest that this cancellation of proprioceptive information disrupts the natural modulation of reciprocal inhibitory networks that is essential to produce alternating recruitment of antagonist motor pools during locomotion. Consequently, only a narrow range of EES parameters can facilitate movement in people with SCI, which is insufficient to enable locomotion without extensive rehabilitation 10,11. Computer simulations guided the identification of EES protocols that not only preserve proprioceptive information but also enable a robust control over 
motoneuron activity. Here, we discuss the significance of these results, stress the dramatic consequences of the transient proprioceptive deafferentation during EES, and envision the avenues for translating these new stimulation protocols clinically.

\section{EES erases proprioceptive information in humans, but not in rats}

Evidence indicates that EES primarily recruits large-diameter afferents within the posterior roots 15. These afferents originate from proprioceptive organs, which sense changes in muscle length and tension, and to a lesser extent, mechanoreceptors within the skin. EES elicits orthodromic action potentials along the recruited afferents that mediate the therapeutic effects of the stimulation18. However, we show that EES also induces antidromic action potentials that travel in the opposite direction. Indeed, recordings of peripheral nerve activity identified antidromic volleys propagating toward sensory organs in response to EES in humans. Previous studies documented the presence of antidromic action potentials traveling along the sensory fibers of the sciatic, peroneal and sural nerves in rats, dogs, nonhuman primates and humans in response to EES applied to thoracic segments1921. Here, we establish the high occurrence of antidromic action potentials when EES targets the lumbar posterior roots.

We reasoned that EES-induced antidromic action potentials may collide with APs conveying proprioceptive information. The annihilation of APs following these collisions is due to the refractory period of Ranvier's nodes. Computer simulations predicted a high occurrence probability of these collisions along the recruited afferents when EES is delivered at frequencies commonly used in human studies to facilitate movements after SCI. Due to the longer length and therefore larger propagation time of APs along human proprioceptive afferents, the incidence of these collisions is considerably higher than in rats. These results suggest that EES may partially cancel proprioceptive information in humans.

To assess this possibility, we conducted experiments that highlighted the consequences of these collisions on the integration of proprioceptive information in the brain and spinal cord of humans. First, we found that the delivery of continuous EES abolishes the conscious perception of leg position and displacement. Second, we showed that proprioceptive information drives the modulation of spinal circuits during movement and the cancellation of this information during continuous EES disrupts this modulation.

Over the past two decades, EES has been applied to thousands of people for pain alleviation, and to improve motor function after SCI8-13,36. For pain treatments, the stimulation is applied at the thoracic level at low intensities. Consequently, there was no obvious loss of sensation in the legs during EES. For SCI, the participants exhibited no or limited sensation in the legs, which may explain why this unexpected cancellation of proprioception information remained unnoticed. However, this phenomenon has far-reaching implications for the development of a therapy to restore locomotion with EES. Indeed, this transient proprioceptive deafferentation not only alters the conscious control of movement and the modulation of spinal circuits with EES, but may also compromise the reorganization of residual descending pathways during rehabilitation enabled by EES. 


\section{Proprioceptive information must be preserved to enable locomotion with EES}

Bipedal locomotion requires the integration of information from a multiplicity of sensory modalities, of which proprioception may be the most important. Proprioceptive information gives rise to a conscious perception of limb positions37 that plays a critical role during walking38,39. For example, the sudden loss of proprioception induces severe gait deficits40,41. Individuals with chronic proprioceptive loss can learn to compensate using other sensory modalities, especially vision 41 . While this adaptation enables them to walk, the associated cognitive load obliges them to rely on a wheelchair for daily life. All our participants reported a loss of limb position awareness during EES. Consequently, this disruption of proprioception strongly limits the clinical relevance of continuous EES to support locomotion during daily living activities in people with SCI.

In addition to its integration in the brain, the information derived from proprioceptive organs is distributed throughout the spinal cord via a dense network of afferent feedback circuits that directly activate motoneurons and shape motor pattern formation during locomotion. Signals from muscle spindles and Golgi tendon organs determine the timing of phase transitions, substantially contribute to leg motoneuron pool recruitment, and coordinate the adaptions of leg movements to unpredictable perturbations and task-specific requirements42-45. Our results suggest that these key mechanisms of motor control are obstructed during continuous EES. Moreover, the interruption of descending pathways reinforces the critical role of these proprioceptive feedback circuits, which become the primary source of control for motor pattern formation46. For example, the integration of proprioceptive information enables the spinal cord to coordinate locomotion across a broad range of speeds, loads and directions in animal models of complete SCI23. The disruption of proprioceptive information during EES would severely deteriorate this ability of the spinal cord to coordinate motor pattern formation after SCI.

We previously documented some of the mechanisms through which EES facilitates locomotion in rats. In particular, we showed that the modulation of reciprocal inhibitory circuits via proprioceptive feedback during each phase of gait directs the excitatory drive elicited by EES towards the motoneuron pools that are functionally relevant at this specific time18. This mechanism transforms the unspecific excitatory drive into a spatially and temporally specific pattern of excitation delivered alternatingly to the motoneuron pools whose activation is required in the flexion and extension phases of the step cycle. The spinal cord thus acts as an elegant filter that endows EES with the necessary specificity for therapeutic applications. Due to the cancellation of proprioceptive information in humans, only narrow ranges of EES frequencies and amplitudes can take advantage of this mechanism. Computer simulations indicate that EES disrupts movement-related modulation of reciprocal inhibitory circuits as soon as the stimulation elicits responses in muscles. The resulting destabilization of the network leads to an imbalance in the excitation of antagonist motor pools, favoring one motor pool over the other. Consequently, the modulation of EES parameters failed to enable the graded control over motoneuron activity that was observed in the rodent computational model. Experimental recordings confirmed these results, both in rodents and humans with SCI. We previously showed that this controllability enables targeting lesion-specific gait deficits and mediating task-specific adjustments of leg 
movements through closed-loop controllers and brain-spine interfaces in rats and nonhuman primates3,5,18. These features may be essential to facilitate the complex postural and propulsive requirements underlying the bipedal gait of humans.

Finally, input from proprioceptive organs plays a determinant role in steering the reorganization of residual descending pathways that helps restore locomotion after SCI. Genetically modified mice lacking functional proprioceptive circuits display defective rearrangements of descending projections after SCI, which abolishes the extensive recovery occurring spontaneously in wild-type mice after the same injury47. Similarly, clinical studies reported that the preservation of proprioceptive information is a key predictor of recovery after neurotrauma48, suggesting that this specific sensory channel may also contribute to steering the reorganization of residual neuronal pathways in humans. Therefore, the disruption of natural proprioception may reduce the ability of EES to augment neuroplasticity and recovery when delivered during rehabilitation.

The multifaceted roles of proprioceptive information for coordinating locomotor functions and steering functional recovery after SCI emphasize the critical importance of identifying EES protocols that preserve proprioceptive information in order to fulfill the therapeutic potential of this treatment paradigm for clinical applications.

\section{EES strategies that replace or preserve proprioceptive information}

We exploited this new understanding to design sensory-compliant EES protocols that circumvent the cancellation of natural proprioception during EES.

We first conceptualized a strategy that aims to replace the cancelled proprioceptive information with a spatiotemporal stimulation profile that encodes the natural firing rates of proprioceptive afferents from each muscle during locomotion. Computer simulations confirmed that this EES protocol not only preserves proprioceptive information but also augments the control over motoneuron activity, while preserving the alternation between antagonist muscles. Realistically, the afferents originating from a single muscle cannot be targeted specifically with current stimulation technologies. However, these stimulation protocols could be approximated with EES bursts delivered over spatially-selective spinal cord regions using a temporal sequence coinciding with the firing profile of the proprioceptive afferents innervating these specific spinal cord regions. This approach shares similarities with EES protocols that encode the spatiotemporal sequence of motoneuron activation during locomotion27. Compared to continuous EES, this targeted stimulation strategy enables a markedly higher degree of control over motoneuron activity in animal models of SCI5,27. The alternation of spatially-selective bursts also preserves the natural proprioceptive information flowing in the dorsal/posterior roots that are not engaged by the stimulation. Our simulations suggest that the delivery of EES bursts should coincide with the profile of proprioceptive afferent firing, which can be partially out of phase with motoneuron activity. However, we believe that this protocol would enhance the control over motoneuron activity and maximize the amount of preserved proprioceptive information. Such a stimulation strategy shares striking similarities with biomimetic approaches developed for the delivery of realistic tactile sensations in human amputees 49 . 
We found that the delivery of EES bursts with a low amplitude, but high frequency, may be an alternative or complementary stimulation strategy to minimize the cancellation of proprioceptive information. Due to the low amplitude, the stimulation recruits a limited number of afferents. Each proprioceptive afferent synapses onto all the homonymous motoneurons31,32. Consequently, the repeated recruitment of these afferents with EES bursts at high frequency leads to a summation of excitatory post-synaptic potentials in motoneurons, which receive an overall amount of excitation equivalent to that induced by continuous EES at high amplitude and low frequency. However, all the non-recruited afferents continue providing essential information about muscle length and tension changes. These results have general implications for EES protocols. First, the modulation of EES bursts allows them to augment the amount of excitation delivered to motoneurons without the need to increase the stimulation amplitude. Second, the lower amplitude requirements would improve the spatial selectivity of the stimulation, since the volume of the electrical field is proportional to the current amplitude.

These novel stimulation protocols require dedicated implantable pulse generators that allow the delivery of EES bursts with high-frequency resolution through independent current sources that are controllable independently in real-time. Various companies are developing next-generation implantable pulse generators that partially meet these requirements.

In parallel, we are conducting a clinical study using a commercially available stimulator that we upgraded to enable real-time control of spatially-selective EES train. We found that within one week, spatiotemporal stimulation enables independent weight-bearing locomotion in the three participants of the present study 50.

These combined findings stress the necessity of developing new neurotechnologies that support the implementation of strategies that preserve proprioception in order to facilitate motor control and steer plasticity with EES in humans.

\section{Materials and Methods}

\section{Computer Simulations}

Computer simulations were performed in python 2.7 using the NEURON51 simulation environment to run the spiking neural network models and OpenSim52 for the biomechanical model of rats and humans. Both the NEURON simulation environment and OpenSim are open-source programs.

Model of a proprioceptive afferent fiber recruited by EES-The afferent fiber model was characterized by two parameters: (i) the propagation time required by an action potential to travel the whole length of the fiber, and (ii) the firing rate at which action potentials are generated by the sensory organ. These parameters were adjusted to meet the properties of all the modeled afferent fibers. For each action potential (AP), we simulated the propagation from the sensory organ of origin to the spinal cord and the refractory dynamics (mean refractory period \pm standard deviation: $1.6 \pm 0.16 \mathrm{~ms}$ ) along the fiber. We modeled EES as a periodic event recruiting the most proximal portion of the fiber. The recruitment only occurred when the fiber was not under refractory period. When a fiber was 
electrically activated, an antidromic AP propagated towards the distal end of the fiber. The encounter of this antidromic AP with a sensory AP traveling towards the spinal cord led to an antidromic collision that cancelled both APs.

Estimation of antidromic collisions probability-The developed fiber model was used to assess the probability of antidromic collisions based on EES frequency, the firing rate of the sensory organs, and the propagation time required by an AP to travel along the whole length of the fiber. Propagation times were set to $2 \mathrm{~ms}$ in rat afferents. Due to the extended length of axons in humans, we modeled human afferents innervating proximal (10 $\mathrm{ms})$ and distal $(20 \mathrm{~ms})$ muscles. Antidromic collision probability was defined as the probability of a natural sensory AP to collide with an EES-induced antidromic AP within a single fiber. For each tested model parameter and stimulation frequency, we integrated the dynamic of the fiber over 60 seconds and evaluated the number of antidromic collisions occurring within this time period. To estimate antidromic collisions probability, we averaged the results of 50 simulations initialized with different EES onset delays varying between 0 and $10 \mathrm{~ms}$.

Rat model of proprioceptive feedback circuits-The rat model of proprioceptive feedback circuits was elaborated from a previously validated model18, which we modified to integrate a simpler and faster model of the motoneurons and the new model of proprioceptive afferents that considers the occurrence of antidromic collisions.

Briefly, this model is composed of four components: (i) a spiking neural network reproducing the proprioceptive feedback circuits associated with a pair of antagonist muscles, (ii) a muscle spindle model, (iii) a musculoskeletal model of the rat hindlimb, and (iv) a finite element method model of EES of the rat lumbar spinal cord (Figure 4A).

The spiking neural network includes populations of group-Ia and group-II afferent fibers, Iainhibitory interneurons, group-II excitatory interneurons, and pools of alpha motoneurons. The number of cells, the number and the strength of the synapses contacting the different populations of neurons, and the characteristics of the cell models are described in our previous work18. To speed up the simulation time, we replaced our previous multicompartmental motoneuron model with an integrate and fire cell model designed to reproduce the realistic membrane response dynamics to excitatory and inhibitory stimuli5356. Specifically, we set the refractory period to $20 \pm 1 \mathrm{~ms}$ and the membrane time constant $\tau_{\text {membrane }}$ to $6 \pm 0.3 \mathrm{~ms}$. Excitatory synapses were modeled as instantaneous changes in current exponentially decaying with time constant $\tau_{\text {excitatory }} 0.25 \mathrm{~ms}$. Inhibitory synapses were modeled as alpha functions with a rise time constant $\tau_{\text {inhibitory_1 }}$ of $2 \mathrm{~ms}$, and a decay time constant $\boldsymbol{\tau}_{\text {inhibitory_2 }}$ of $4.5 \mathrm{~ms}$ (Supplementary Figure $7 \mathrm{a}$ ). We adjusted the motoneurons synaptic weights to match experimental excitatory and inhibitory post-synaptic potentials (EPSPs/IPSPs). For this, we normalized experimental EPSPs54,55 and IPSPs56 to the minimum depolarization necessary to induce an AP in our multicompartmental model (Supplementary Figure 7b,c). Afferent fibers were modeled with an AP propagation time of $2 \mathrm{~ms}$. This parameter was estimated to represent rat afferent fibers innervating the antagonist muscles of the ankle. 
The musculoskeletal57,58 and muscle spindle 30 models were used to calculate the firing rate profiles of group-Ia and group-II afferent fibers innervating the flexor (tibialis anterior) and extensor (gastrocnemius medialis) muscles of the ankle during locomotion. For this purpose, we steered the musculoskeletal model with previously obtained recordings of the rat hindlimb kinematics during locomotion to estimate the ankle muscles stretch profiles through inverse kinematics. We then used the muscle spindle model to compute the firing rate profiles. To mimic the alpha-gamma linkage, muscles stretch and stretch velocity were linked to the envelope of EMG activity from the homonymous muscle (Equations 1 and 230). The estimated afferent firing rate profiles drove the activity of the modeled proprioceptive afferents.

A validated finite element method model of EES of the lumbar spinal cord 15 was finally used to estimate the proportion of afferent and efferent fibers recruited at a given stimulation amplitude. Realistic interactions between EES and the natural sensory activity along the modeled afferent fibers were integrated using the developed proprioceptive afferent model.

$$
\begin{aligned}
& \text { Ia firing rate }=50+2 \cdot \text { stretch }+4.3 \cdot \operatorname{sign}(\text { stretchVelocity }) \cdot \mid \text { stretchVelocity }\left.\right|^{0.6}+50 \\
& \cdot E M G_{\text {env }}
\end{aligned}
$$

\section{(Equation 1)}

$$
\text { II firing rate }=80+13.5 \cdot \text { stretch }+20 \cdot E M G_{\text {env }} \quad \text { (Equation 2) }
$$

Human model of proprioceptive feedback circuits-The layout of the rat model served as a basis to build the human model of proprioceptive feedback circuits. To take into account the specific anatomical and physiological features of humans, we adapted the musculoskeletal model, the muscle spindle model, the weights of the synapses in the network, the length of the modeled afferent fibers, and the output of the finite element method model of EES (Figure 4a).

To estimate the stretch of flexor (tibialis anterior) and extensor (soleus) muscles spanning the ankle joints, we used the 3DGaitModel2392 OpenSim lower limb model59 and kinematic data of healthy subjects during locomotion on a treadmill28,60. We tuned the muscle spindle model to account for the lower firing rates of human proprioceptive afferents compared to those of rodents22,61. Specifically, we scaled Equations 1 and 2 down by 0.2 and 0.25 , respectively, to produce firing rates that remained within the range of values generally observed in humans (rarely exceeding 30 Impulse/second22,30,62). The envelopes of EMG activity were extracted from the same subjects from whom we also extracted the kinematic data28,60.

We assumed that if the occurrence probability of antidromic collisions would be the same in humans and rodents, the human model should reproduce results that are qualitatively similar 
to the simulations obtained in rats. Hence, we optimized the weight of the synaptic connections between the afferent fibers and their target spinal neurons by driving the network with the estimated human afferent firings but without modifying the propagation time required by sensory APs to reach the spinal cord - a parameter proportional to the occurrence probability of antidromic collisions (Supplementary Figure 8a). To this purpose we performed a systematic search by progressively increasing the synaptic weights of connections from afferent fibers. EES frequency and percentage of Ia-afferents recruited by EES were set to $60 \mathrm{~Hz}$ and to $60 \%$, respectively. We defined a set of fitness functions and relative minimum scores to define the range of synaptic weights that produce the desired behavior of the network (Equation 3) and selected one set of weights for further simulations (Supplementary Figure 8b,c).

$$
\left\{\begin{array}{l}
\text { percentile_90 }\left(\text { Motoneurons } \boldsymbol{F} \boldsymbol{R}_{\text {ext }}\right)>5 \mathrm{Imp} / \mathrm{s} \\
\text { percentile_90 }\left(\text { MotoneuronsF } \boldsymbol{R}_{\text {flex }}\right)>5 \mathrm{Imp} / \mathrm{s} \\
1-\text { mean }\left(\text { Motoneurons } \boldsymbol{F} \boldsymbol{R}_{\text {flex }} \cdot \text { Motoneurons } \boldsymbol{F} \boldsymbol{R}_{\text {ext }}\right)>0.9
\end{array} \quad\right. \text { (Equation 3) }
$$

We then modified the AP propagation time parameter of the afferent fiber models to $16 \mathrm{~ms}$, which is a representative value for the proprioceptive afferents of the ankle muscles in humans63.

We assumed that the ratio between the amount of primary and secondary afferent fibers recruited by EES while increasing the stimulation amplitude is similar in rats and humans. We thus used the finite element method model of the rat spinal cord to estimate the percentage of primary and secondary afferents recruited by the stimulation. However, to take into account the considerably larger distance of the ventral roots from the epidural electrodes, we did not simulate the direct recruitment of motor axons. This phenomenon commonly occurs in rats but is limited in humans 14,15 . While this decision was taken in order to build a more realistic model, simulating the direct recruitment of motor axons as in the rat model would have not influenced the significance of the presented results. Indeed, given the low amplitudes tested in this work, only $7 \%$ of the simulated rat motoneuron axons were recruited directly by EES at the highest stimulation amplitude tested (Figure 5a).

Spatiotemporal stimulation profiles-Spatiotemporal EES profiles encoding the natural proprioceptive information originating from a pair of antagonist muscles spanning the ankle joint were estimated in two steps. First, we computed the normalized average firing rate profiles of group-Ia, group-II and group-Ib afferents over a gait cycle. Second, these three profiles were averaged to produce a stimulation profile that encodes the global proprioceptive information (Figure 7a). Since group-Ib afferent firing is closely correlated to the activity of the muscle along which the associated Golgi tendon organ is connected30, we approximated the firing rates of group-Ib afferents with the envelope of the EMG activity from the homonymous muscle during gait. Simulations were conducted using the estimated stimulation profile for each muscle. EES amplitude was adjusted proportionally to the changes in the estimated stimulation profile while the length of the stimulation profile was adjusted based on the duration of each gait cycle. 
High-frequency low-amplitude EES model-To assess the effect of high-frequency low-amplitude EES on the membrane potentials of motoneurons, we used our previously validated multicompartmental motoneuron model that integrates realistic synaptic boutons from group-Ia afferents18 (Figure 8a,b). However, simulations on the effect of highfrequency low-amplitude EES on the muscle spindle feedback circuits were still performed using the simplified integrate and fire motoneuron model (Supplementary Figure 7). The more realistic multicompartmental model was used in order to obtain a more accurate estimate of motoneurons' soma responses to high-frequency bursts of EES.

Limitations of the human computational model-Microneurographic recordings of group-Ia and group-II afferents during slow movements reported that firing rates rarely exceed $30 \mathrm{Imp} / \mathrm{s}$ in humans22,64,65. In the human computational model, we thus limited muscle spindle firing to $50 \mathrm{Imp} / \mathrm{s}$ during gait, which is markedly lower than peak firings of up to $200 \mathrm{Imp} / \mathrm{s}$ reported during locomotion in quadrupedal mammals. Nevertheless, we cannot exclude the possibility that human muscle spindle afferents fire at higher rates during gait. Indeed, locomotion involves higher movement speeds than those commonly used during microneurographic recordings in humans. Consequently, the actual range of firing rates underlying the activity of group-Ia fibers during human gait remains unknown. While higher firing rates might affect the predictions of our model, the overall conclusions would remain unchanged, since EES would still block a significant amount of proprioceptive information for high firing rates. Therefore, the degree of disruption may scale with the actual range of afferent firings, but the conclusion derived from this model would still hold.

\section{Experimental Procedures in Humans}

\section{Spinal cord stimulation system implanted in human subjects with $\mathrm{SCl}-$}

Experiments conducted in human subjects with SCI were carried out within the framework of an ongoing clinical study (ClinicalTrials.gov Identifier: NCT02936453) that has been approved by Swiss authorities (Swissethics protocol number 04/2014 ProjectID: PB_2016-00886, Swissmedic protocol 2016-MD-0002), and were in compliance with all relevant clinical regulations. The study is conducted at the Lausanne University Hospital (CHUV). All subjects signed written inform consent prior to their participation. The subjects were surgically implanted with a spinal cord stimulation system comprising an implantable pulse generator (Activa ${ }^{\mathrm{TM}} \mathrm{RC}$, Medtronic plc, Fridley, Minnesota, SA) connected to a 16electrode paddle array (Medtronic Specify ${ }^{\mathrm{TM}}$ 5-6-5 surgical lead) that was placed over the lumbosacral segments of the spinal cord. Subject related data and details on their neurological status at their entry into the clinical study, evaluated according to the International Standards for Neurological Classification of Spinal Cord Injury, are provided in the Life Sciences Reporting Summary and in Supplementary Table 1. Subjects' recruitment process is described in the Life Sciences Reporting Summary.

\section{Recording of EES-induced antidromic activity along human afferents-}

Recordings of the neural activity induced by EES were performed with the NIM Eclipse system (Medtronic plc, Fridley, Minnesota, USA). The activity of the soleus muscle was recorded with surface EMG electrodes (Ambu Neuroline 715, Ambu Sarl, Bordeaux, France), while the activity of the sural and of the proximal and distal branches of the tibial 
nerve were recorded using percutaneous disposable needle electrodes (Ambu Neuroline Twisted Pair Subdermal 12 x 0.4 mm, Ambu Sarl, Bordeaux, France). The proximal branch of the tibial nerve was recorded at the level of the popliteal fossa (Figure 2a). The recording needle electrode insertion point was at the site that elicited an H-reflex at the lowest stimulation amplitude, identified by using a stimulation probe. The distal branch of the tibial nerve was recorded at the level of the medial malleolus (Figure 2a). The recording electrode position was determined by applying electrical stimulation to this site and by verifying the evoked potentials at the level of the proximal branch of the tibial nerve. The sural nerve was recorded at the level of the lateral malleolus. The specific location of the electrode was defined following the same procedure as for the distal branch of the tibial nerve. Neural and EMG signals were sampled at $10000 \mathrm{~Hz}$, amplified, and band-pass filtered $(30-1000 \mathrm{~Hz})$ online. For the entire duration of the experiment, participants remained relaxed in a supine position. EES was delivered at $20 \mathrm{~Hz}$ for 60 seconds in order to collect a total of approximately 1200 pulses. We selected EES sites that mainly recruited the posterior root innervating the S1 spinal segment, as verified in the presence of reflex responses in the soleus muscle following each pulse of EES. For the experiment, the stimulation amplitude was reduced until no muscle contraction was noticeable to avoid contaminating neural recordings with electromyographic activity or movement artifacts. To verify that the stimulation amplitude was sufficient to recruit afferent fibers in the recorded nerves, we controlled that the stimulation elicited a sensation of tingling in the corresponding dermatome. We recorded EES artifacts with surface electrodes positioned over the vertebral levels of the implanted paddle array. The artifacts were used as triggers to extract and average the evoked potentials.

Assessment of proprioceptive function during EES-The threshold to detection of passive movement test66 was performed with the Humac Norm Cybex system (Computer Sports Medicine Inc., Stoughton, US). Subjects were first tested without EES and then during continuous EES. Throughout the experiment, participants' tactile, visual, and aural information were occluded by using foam cushions, blindfolds, and headphones with pink noise. The experimental protocol was tailored for each participant, since each of them presented distinct levels of residual proprioceptive functions. At the beginning of each trial, the participant's knee joint was moved to an initial position of 45 degrees of extension. The participant was informed with a tap on the shoulder that a new trial was about to start. The trial was then started after a randomised time delay to assess false positive detections. In subject \#1, we imposed movements of knee extension or knee flexion from the initial position at a constant angular velocity of 0.5 degrees per second. Flexion and extension were delivered randomly. The participant was instructed to report the movement direction, as soon as he became aware of it, by pushing a button. A maximum displacement of 15 degrees was allowed (Figure 2b). Button-triggered digital signal and joint kinematics were recorded at a sampling frequency of $5000 \mathrm{~Hz}$. The trial was considered successful if the direction of the movement was correctly identified. A trial was considered unsuccessful when the movement was either misclassified or not perceived at all within the limited range of movement. Subject \#3 was not able to detect the direction of the imposed movement, even in the absence of continuous EES. To simplify the task, we limited the movement to knee extension only, increased the movement speed to 1 degree per second, and allowed a 
maximum displacement of 30 degrees (Figure 2b). A trial was considered successful if the movement was detected within the allowed range of movement. Subject \#2 was not able to perceive the imposed movements and was thus excluded from this experiment.

A minimum of 10 repetitions were performed to complete an assessment for a given EES condition. The proportion between successful and unsuccessful trials was used to compute participants' error rate and 95\% confidence interval by using the Clopper-Pearson interval method based on Beta distribution.

We adjusted the configuration of EES electrodes to target both flexor and extensor muscles of the knee. Recordings of the EMG activity from the vastus lateralis and semitendinosus muscles allowed the identification of the minimum stimulation amplitude necessary to recruit these muscles. We then assessed the proprioceptive functions of the subjects during continuous EES that was delivered with amplitudes below ( 0.8 times) and above ( 1.5 times) the muscle response threshold. For both amplitudes, we tested a range of frequencies: 10, 30, 50 and $100 \mathrm{~Hz}$. At 1.5 the muscle response threshold amplitude, frequencies below 50 $\mathrm{Hz}$ induced spastic contractions, and were thus not tested. The sequence of the tested stimulation parameters was randomized.

\section{Assessment of EES-induced responses modulated during passive joint} movements-The Humac Norm Cybex was used to impose passive joint movements with a sinusoidal profile of fixed amplitude and frequency, while continuous EES was delivered to produce motor responses in the muscles spanning this joint. The subjects were asked to relax, neither to resist, follow, nor facilitate the movements. Muscle responses and EES artifacts were recorded with wireless surface EMG electrodes (Myon 320, Myon AG, Schwarzenberg, Switzerland) at a sampling frequency of $5000 \mathrm{~Hz}$. Joint kinematics was recorded with the Cybex system at $5000 \mathrm{~Hz}$. EES parameters, as well as the targeted joint, the angular velocity and the amplitude of the movement were set depending on subjectspecific constraints (Figure 3a and Supplementary Figure 2). In subject \#1, the Cybex system was used to produce flexion and extension movements of the ankle joint at a frequency of $1.13 \mathrm{~Hz}$ and a range of motion of 30 degrees. These parameters were chosen to be as large as possible in order to maximize the amount of proprioceptive signals generated from the targeted muscles while minimizing discomfort. EES electrodes were configured in order to recruit the targeted muscles. EES was delivered with frequencies ranging from 5 to $60 \mathrm{~Hz}$, presented in a random order. The stimulation amplitude was set to induce consistent muscle responses across the range of tested frequencies, corresponded to 1.25 times the muscle response threshold. For each condition tested, a minimum of 1 minute of recording was performed. Recording duration was extended to 2 minutes when EES was delivered at 5 Hz. In subject \#2 and \#3, we could not find electrode configurations that recruited the targeted muscles without causing discomfort at the required EES amplitudes and frequencies. Therefore, we adapted the experiment and targeted the knee joint instead of the ankle joint. Moreover, we limited the range of tested frequencies. Specifically, for subject \#3 we kept an oscillation frequency of $1.13 \mathrm{~Hz}$, set a movement range of 60 degrees, and limited the range of EES frequencies from 10 to $60 \mathrm{~Hz}$. These settings also led to spastic contractions in subject \#2. Consequently, we reduced the movement range and frequency to 
50 degrees and $0.9 \mathrm{~Hz}$, respectively, and limited the range of EES frequencies between 20 and $60 \mathrm{~Hz}$.

To quantify the modulation of muscle responses during the passive movements, we extracted the timing of each EES pulse with the recorded stimulation artifacts. We then extracted the muscles responses and grouped them according to the phase of the cyclic movement $(\mathrm{n}=10$ bins) (Figure 3b). When more than one EES pulse occurred within a given bin, only the response with highest amplitude was selected. We bootstrapped the normalized modulation depth median and $95 \%$ confidence interval (Equation 4) by computing the median peak to peak amplitudes $(m P 2 P s)$ of the responses occurred in the different bins. Normalization was performed in order to account for frequency-dependent depression of EES-induced muscle responses67-69.

$$
\text { NormalizedModulationDepth }=\frac{[\max (m P 2 P s)-\min (m P 2 P s)]}{\min (m P 2 P s)} \quad \text { (Equation 4) }
$$

Continuous EES during locomotion on a treadmill-The FLOAT robotic suspension system (Lutz Medical Engineering AG, Rudlingen, Switzerland) was used to provide the participants with personalized upward and forward forces to the trunk during locomotion on a treadmill28,70. EES was delivered through four independent configurations of electrodes. Each configuration involved one or multiple anodes and cathodes. We configured these electrode combinations to target the left and right posterior roots projecting to the L1 and L4 segments. For this purpose, we searched the electrode configurations that activated preferentially the iliopsoas and the tibialis anterior. These motor pools spanned the L1/L2 segments and L4/L5 segments, respectively. The amplitude and frequency of these four electrode configurations were optimized by visual inspection of the induced EMG activity and facilitation of kinematics when subjects were asked to step on the treadmill. Different EES frequencies and amplitudes were tested to characterize the ability of EES to modulate the motor output. The order of the tested parameters was randomized. EMG recordings were performed with wireless surface electrodes (Myon 320, Myon AG, Schwarzenberg, Switzerland) and recorded at $1000 \mathrm{~Hz}$. Leg kinematics was recorded using the Vicon motion capture system (Vicon Motion Systems, Oxford, UK) at $100 \mathrm{~Hz}$. Subjects were allowed to use the handrails of the treadmill to facilitate their leg movements. Analysis of EMG activity and kinematics was conducted using methods reported in details previously 28 .

\section{Electrophysiological recordings of high-frequency low-amplitude EES-EES} was delivered through electrode configurations that were used to facilitate locomotion. Motor responses to both single pulses and bursts of 4 pulses at $500 \mathrm{~Hz}$ were recorded from different lower limb muscles with wireless surface electrodes at a sampling rate of $5000 \mathrm{~Hz}$ (Myon 320, Myon AG, Schwarzenberg, Switzerland). The responses of the muscle that was recruited the most were used for the analyses. During the experiment, the participants were in the supine position. 


\section{Experimental Procedures in Rats}

Animal husbandry-All procedures and surgeries were approved by the Veterinary Office of the canton of Geneva in Switzerland, and were in compliance with all relevant ethical regulations. The experiments were conducted in 9,11-week-old, female Lewis rats ( $\sim 220 \mathrm{~g}$ body weight) and 4, 11-week-old, Long-Evans rats ( $\sim 240 \mathrm{~g}$ body weight). Rats were housed separately with a light/dark cycle of 12 hours.

Surgical procedures-Surgical procedures have been described in detail previously2,23. All the interventions were performed in aseptic conditions and under general anesthesia. Briefly, rats received a severe thoracic (T8) contusion SCI (250 kdyn) by using a forcecontrolled spinal cord impactor (IH-0400 Impactor, Precision Systems and Instrumentation LLC, USA). During the same surgery, EES electrodes were sutured to the dura overlying the midline of S1 and L2 spinal segments in Lewis rats, and of L4 and L2 spinal segments in Long-Evans rats. Electrodes were created by removing a small part of insulation $(\sim 400 \mu \mathrm{m})$ from Teflon-coated stainless-steel wires (AS632, Cooner Wire, USA). A common ground wire electrode ( $\sim \mathrm{cm}$ of active site) was placed subcutaneously over the right shoulder. Finally, bipolar electrodes (same type as used for EES) were implanted bilaterally in the left and right tibialis anterior muscles to record the EMG activity.

\section{Assessment of EES induced responses modulated during passive joint} movements-Lewis rats ( $\mathrm{n}=4$ ) were lightly anesthetized (Ketamine: $75 \mathrm{mg} / \mathrm{kg}$ and Xylazine $5 \mathrm{mg} / \mathrm{kg}$, ip) and positioned in a prone position within a support system that let the hindlimbs hanging freely. The right paw was secured within a 3D printed pedal connected to a stepper motor (QSH4218-51-10-049, Trinamic Motion Control GmbH, Waterloohain, Germany). We used this robotic platform to impose cyclic movements of the ankle with a fixed amplitude (70 degrees) and frequency $(0.54 \mathrm{~Hz})$, while continuous EES was delivered to evoke responses in the tibialis anterior muscle (Figure 3e). EES was delivered using an IZ2H Stimulator controlled by a RZ2 BioAmp Processor (Tucker-Davis Technologies, Alachua, US). EES amplitude was set to approximately 1.2 times the muscle response threshold. We tested EES frequencies ranging from 5 to $100 \mathrm{~Hz}$, delivered in a random order. EMG activity of the tibialis anterior was amplified with a PZ3 Low Impedance Amplifier (Tucker-Davis Technologies, Alachua, US) and recorded with the RZ2 BioAmp Processor at a sampling frequency of $24414 \mathrm{~Hz}$. Ankle kinematics was record with the Vicon motion capture system at sampling frequency of $200 \mathrm{~Hz}$. For each tested EES condition a minimum of 1 minute of recording was performed. To analyze the modulation of the muscle responses, we used the same procedures that we adopted in the equivalent experiment carried out in human subjects.

\section{Electrophysiological recordings of high-frequency low-amplitude EES-We} tested the impact of high-frequency low-amplitude EES in 5 Lewis rats. EES and EMG recordings were performed with the setup used for assessing the modulation of muscle responses during passive movements. The muscle response threshold was measured using single pulses of EES that were delivered at amplitudes close to this threshold, thus allowing to obtain a precise value. High-frequency bursts were then delivered at amplitudes below the identified motor response threshold. The aim was to evaluate whether high-frequency 
stimulation was able to recruit trans-synaptically motoneurons at lower amplitudes than single pulses. For each amplitude, we tested burst frequencies ranging from 100 to $1000 \mathrm{~Hz}$. The duration of each burst was kept constant at $25 \mathrm{~ms}$. During the experiments, the rats were held in a resting position with the hindlimbs hanging freely.

Continuous EES during locomotion on a treadmill-Behavioural experiments during locomotion were performed in 4 Long-Evans rats. Following one to two weeks of rehabilitation using previously described procedures 2,23 , we evaluated the impact of different EES frequencies on the modulation of muscle activity and hindlimb kinematics during bipedal locomotion on a treadmill. Locomotion was recorded without EES and with EES at frequencies ranging from 20 to $80 \mathrm{~Hz}$, delivered in a random order. EES amplitude was kept fixed at the optimal value found during training. For each experimental condition, approximatively 10 gait cycles or 20 seconds of minimal leg movements were recorded.

Hindlimb kinematics was recorded with the Vicon motion capture system (Vicon Motion Systems, Oxford, UK) at a sampling frequency of $200 \mathrm{~Hz}$. EMG signals were amplified and filtered online (10-10000 Hz band-pass) by a Differential AC Amplifier (A-M System, Sequim, US) and recorded at $2000 \mathrm{kHz}$ with the Vicon system.

Statistics-No statistical methods were used to pre-determine sample sizes but our sample sizes are similar to those reported in previous publications using similar experimental procedures $13,15,18,66$. Data collection and analysis were not performed blind to the conditions of the experiments. No data were excluded from the analyses. Different EES conditions were tested on the same animals or participants, and thus no control groups were used. In each experiment, the order of the tested EES conditions was randomized as described in the relevant Methods sections and in the Life Science Reporting Summary. Data are reported as mean \pm standard error of the mean (SEM.) or median values $\pm 95 \%$ confidence interval (CI.). Confidence intervals and significance were analyzed using the non-parametric Wilcoxon rank-sum two-sided test with Bonferroni correction for multiple comparisons, two-tailed Wald test, the Clopper-Pearson interval based on Beta distribution method, or a bootstrapping approach based on the Monte Carlo algorithm resampling scheme ( $\mathrm{n}=10,000$ iterations). Linear regression between step height and EES frequency (Figure 6c) was performed assuming a normal distribution of the residuals around zero, however this was not formally tested. No other assumptions were performed.

\section{Supplementary Material}

Refer to Web version on PubMed Central for supplementary material.

\section{Acknowledgments}

We thank Kay Bartholdi, Arnaut Bichat and Laetitia Baud for their help with the rat experiments, and all the individuals involved in the STIMO clinical study. This research was supported by the HBP Neurorobotics Platform funded from the European Union's Horizon 2020 Framework Programme for Research and Innovation under the Specific Grant Agreement No. 720270 (Human Brain Project SGA1).

Funding 
Financial support was provided by the European Union's Horizon 2020 Framework Programme for Research and Innovation under the Specific Grant Agreement No. 720270 (Human Brain Project SGA1) and No. 785907 (Human Brain Project SGA2); RESTORE: Eurostars E10889, Wings for life, GTXmedical, Consolidator Grant from the European Research Council [ERC-2015-CoG HOW2WALKAGAIN 682999], National Center of Competence in Research (NCCR) Robotics of the Swiss National Science Foundation, the Commission of Technology and Innovation (CTI) Innosuisse (CTI) OptiStim 25761.1, International Foundation for Research in Paraplegia (IRP), the Michel-Adrien Voirol Foundation, the Firmenich Foundation, the Pictet Group Charitable Foundation, the Panacée Foundation and the Marie-Curie EPFL fellowship program.

\section{References}

1. Kiehn O. Decoding the organization of spinal circuits that control locomotion. Nature Publishing Group. 2016; :1-15. DOI: 10.1038/nrn.2016.9

2. Van den Brand R, et al. Restoring Voluntary Control of Locomotion after Paralyzing Spinal Cord Injury. Science. 2012; 336:1182-1185. [PubMed: 22654062]

3 . Wenger N, et al. Closed-loop neuromodulation of spinal sensorimotor circuits controls refined locomotion after complete spinal cord injury. Science Translational Medicine. 2014; 6:255ra133$255 \mathrm{ra} 133$.

4. Musienko P, et al. Somatosensory control of balance during locomotion in decerebrated cat. J Neurophysiol. 2012; 107:2072-2082. [PubMed: 22236709]

5. Capogrosso M, et al. A brain-spine interface alleviating gait deficits after spinal cord injury in primates. Nature Publishing Group. 2016; 539:284-288.

6. Asboth L, et al. Cortico-reticulo-spinal circuit reorganization enables functional recovery after severe spinal cord contusion. Nat Neurosci. 2018; 21:576-588. [PubMed: 29556028]

7. Dimitrijevic MR, Gerasimenko YP, Pinter MM. Evidence for a spinal central pattern generator in humans. Ann N Y Acad Sci. 1998; 860:360-376. [PubMed: 9928325]

8. Minassian K, et al. Stepping-like movements in humans with complete spinal cord injury induced by epidural stimulation of the lumbar cord: electromyographic study of compound muscle action potentials. Spinal Cord. 2004; 42:401-416. [PubMed: 15124000]

9. Herman R, He J, D'Luzansky S, Willis W, Dilli S. Spinal cord stimulation facilitates functional walking in a chronic, incomplete spinal cord injured. Spinal Cord. 2002; 40:65-68. [PubMed: 11926417]

10. Angeli C, et al. Recovery of over-ground walking after chronic motor complete spinal cord Injury. N Engl J Med. 2018; doi: 10.1056/NEJMoa1803588

11. Gill ML, et al. Neuromodulation of lumbosacral spinal networks enables independent stepping after complete paraplegia. Nature Medicine. 2018; 377:1938.

12. Harkema SJ, et al. Effect of epidural stimulation of the lumbosacral spinal cord on voluntary movement, standing, and assisted stepping after motor complete paraplegia: a case study. The Lancet. 2011; 377:1938-1947.

13. Angeli CA, Edgerton VR, Gerasimenko YP, Harkema SJ. Altering spinal cord excitability enables voluntary movements after chronic complete paralysis in humans. Brain. 2014; 137:1394-1409. [PubMed: 24713270]

14. Rattay F, Minassian K, Dimitrijevic MR. Epidural electrical stimulation of posterior structures of the human lumbosacral cord: 2. quantitative analysis by computer modeling. Spinal Cord. 2000; 38:473-489. [PubMed: 10962608]

15. Capogrosso M, et al. A computational model for epidural electrical stimulation of spinal sensorimotor circuits. J Neurosci. 2013; 33:19326-19340. [PubMed: 24305828]

16. Gerasimenko YP, et al. Spinal cord reflexes induced by epidural spinal cord stimulation in normal awake rats. 2006; 157:253-263.

17. Minassian K, et al. Human lumbar cord circuitries can be activated by extrinsic tonic input to generate locomotor-like activity. Hum Mov Sci. 2007; 26:275-295. [PubMed: 17343947]

18. Moraud EM, et al. Mechanisms Underlying the Neuromodulation of Spinal Circuits for Correcting Gait and Balance Deficits after Spinal Cord Injury. Neuron. 2016; 89:814-828. [PubMed: 26853304] 
19. Su CF, Haghighi SS, Oro JJ, Gaines RW. 'Backfiring' in spinal cord monitoring. High thoracic spinal cord stimulation evokes sciatic response by antidromic sensory pathway conduction, not motor tract conduction. Spine. 1992; 17:504-508. [PubMed: 1621148]

20. Hunter JP, Ashby P. Segmental effects of epidural spinal cord stimulation in humans. J Physiol (Lond.). 1994; 474:407-419. [PubMed: 8014902]

21. Buonocore M, Bonezzi C, Barolat G. Neurophysiological evidence of antidromic activation of large myelinated fibres in lower limbs during spinal cord stimulation. Spine. 2008; 33:E90-3. [PubMed: 18277861]

22. Prochazka A. Proprioceptive feedback and movement regulation. Handbook of physiology. 1996; 76:125.

23. Courtine G, et al. Transformation of nonfunctional spinal circuits into functional states after the loss of brain input. Nat Neurosci. 2009; 12:1333-1342. [PubMed: 19767747]

24. Capaday C, Stein RB. Amplitude modulation of the soleus H-reflex in the human during walking and standing. Journal of Neuroscience. 1986; 6:1308-1313. [PubMed: 3711981]

25. Courtine G, Harkema SJ, Dy CJ, Gerasimenko YP, Dyhre-Poulsen P. Modulation of multisegmental monosynaptic responses in a variety of leg muscles during walking and running in humans. J Physiol (Lond.). 2007; 582:1125-1139. [PubMed: 17446226]

26. Dy CJ, et al. Phase-dependent modulation of percutaneously elicited multisegmental muscle responses after spinal cord injury. J Neurophysiol. 2010; 103:2808-2820. [PubMed: 20357075]

27. Wenger N, et al. Spatiotemporal neuromodulation therapies engaging muscle synergies improve motor control after spinal cord injury. Nature Medicine. 2016; 22:138-145.

28. Mignardot J-B, et al. A multidirectional gravity-assist algorithm that enhances locomotor control in patients with stroke or spinal cord injury. Science Translational Medicine. 2017; 9

29. Conway BA, Hultborn H, Kiehn O. Proprioceptive input resets central locomotor rhythm in the spinal cat. Exp Brain Res. 1987; 68

30. Prochazka A. Quantifying proprioception. Prog Brain Res. 1999; 123:133-142. [PubMed: 10635710]

31. Mendell LM, Henneman E. Terminals of single Ia fibers: location, density, and distribution within a pool of 300 homonymous motoneurons. Journal of Neurophysiology. 1971; 34:171-187. [PubMed: 5540577]

32. Segev I, Fleshman JW, Burke RE. Computer simulation of group Ia EPSPs using morphologically realistic models of cat alpha-motoneurons. Journal of Neurophysiology. 1990; 64:648-660. [PubMed: 2213137]

33. Collins WF, Honig MG, Mendell LM. Heterogeneity of group Ia synapses on homonymous alphamotoneurons as revealed by high-frequency stimulation of Ia afferent fibers. Journal of Neurophysiology. 1984; 52:980-993. [PubMed: 6512594]

34. Koerber HR, Mendell LM. Modulation of synaptic transmission at Ia-afferent connections on motoneurons during high-frequency afferent stimulation: dependence on motor task. Journal of Neurophysiology. 1991; 65:1313-1320. [PubMed: 1651999]

35. Bawa P, Chalmers G. Responses of human motoneurons to high-frequency stimulation of Ia afferents. Muscle Nerve. 2008; 38:1604-1615. [PubMed: 19016548]

36. Carhart MR, He J, Herman R, D'Luzansky S, Willis WT. Epidural spinal-cord stimulation facilitates recovery of functional walking following incomplete spinal-cord injury. IEEE Trans Neural Syst Rehabil Eng. 2004; 12:32-42. [PubMed: 15068185]

37. Proske U, Gandevia SC. The proprioceptive senses: their roles in signaling body shape, body position and movement, and muscle force. Physiol Rev. 2012; 92:1651-1697. [PubMed: 23073629]

38. Dietz V. Proprioception and locomotor disorders. Nat Rev Neurosci. 2002; 3:781-790. [PubMed: 12360322]

39. Tuthill JC, Azim E. Proprioception. Current Biology. 2018; 28:R194-R203. [PubMed: 29510103]

40. Sanes JN, Mauritz KH, Dalakas MC, Evarts EV. Motor control in humans with large-fiber sensory neuropathy. Hum Neurobiol. 1985; 4:101-114. [PubMed: 2993208]

41. Cole J. Pride and a Daily Marathon. MIT Press; 1995. 
42. Dietz V, Duysens J. Significance of load receptor input during locomotion: a review. Gait \& Posture. 2000; 11:102-110. [PubMed: 10899663]

43. Rossignol S, Dubuc R, Gossard J-P. Dynamic sensorimotor interactions in locomotion. Physiol Rev. 2006; 86:89-154. [PubMed: 16371596]

44. Hultborn H, Nielsen JB. Spinal control of locomotion--from cat to man. Acta Physiol (Oxf). 2007; 189:111-121. [PubMed: 17250563]

45. Prochazka A, Yakovenko S. Predictive and reactive tuning of the locomotor CPG. Integr Comp Biol. 2007; 47:474-481. [PubMed: 21672856]

46. Edgerton VR, Tillakaratne NJK, Bigbee AJ, de Leon RD, Roy RR. Plasticity of the spinal neural circuitry after injury. Annu Rev Neurosci. 2004; 27:145-167. [PubMed: 15217329]

47. Takeoka A, Vollenweider I, Courtine G, Arber S. Muscle Spindle Feedback Directs Locomotor Recovery and Circuit Reorganization after Spinal Cord Injury. Cell. 2014; 159:1626-1639. [PubMed: 25525880]

48. Park S-W, Wolf SL, Blanton S, Winstein C, Nichols-Larsen DS. The EXCITE Trial: Predicting a clinically meaningful motor activity log outcome. Neurorehabil Neural Repair. 2008; 22:486-493. [PubMed: 18780883]

49. Saal HP, Bensmaia SJ. Biomimetic approaches to bionic touch through a peripheral nerve interface. Neuropsychologia. 2015; 79:344-353. [PubMed: 26092769]

50. Wagner F, et al. Targeted neurotechnologies restore walking in humans with spinal cord injury. Nature. 2018

51. Hines ML, Carnevale NT. The NEURON simulation environment. Neural Comput. 1997; 9:11791209. [PubMed: 9248061]

52. Delp SL, et al. OpenSim: open-source software to create and analyze dynamic simulations of movement. Biomedical Engineering, IEEE Transactions on. 2007; 54:1940-1950.

53. Burke RE. Group Ia synaptic input to fast and slow twitch motor units of cat triceps surae. J Physiol (Lond.). 1968; 196:605-630. [PubMed: 5664234]

54. Munson JB, Fleshman JW, Sypert GW. Properties of single-fiber spindle group II EPSPs in triceps surae motoneurons. Journal of Neurophysiology. 1980; 44:713-725. [PubMed: 6448918]

55. Harrison PJ, Taylor A. Individual excitatory post-synaptic potentials due to muscle spindle Ia afferents in cat triceps surae motoneurones. J Physiol (Lond.). 1981; 312:455-470. [PubMed: 6455515]

56. McIntyre CC, Grill WM. Extracellular stimulation of central neurons: influence of stimulus waveform and frequency on neuronal output. Journal of Neurophysiology. 2002; 88:1592-1604. [PubMed: 12364490]

57. Johnson WL, Jindrich DL, Roy RR, Edgerton VR. A three-dimensional model of the rat hindlimb: musculoskeletal geometry and muscle moment arms. J Biomech. 2008; 41:610-619. [PubMed: 18061600]

58. Johnson WL, Jindrich DL, Zhong H, Roy RR, Edgerton VR. Application of a rat hindlimb model: a prediction of force spaces reachable through stimulation of nerve fascicles. IEEE Trans Biomed Eng. 2011; 58:3328-3338. [PubMed: 21244999]

59. Delp SL, et al. An interactive graphics-based model of the lower extremity to study orthopaedic surgical procedures. Biomedical Engineering, IEEE Transactions on. 1990; 37:757-767.

60. Wojtusch J, von Stryk O. HuMoD - A versatile and open database for the investigation, modeling and simulation of human motion dynamics on actuation level. IEEE; 74-79.

61. Hník P, Lessler MJ. Changes in muscle spindle activity of the chronically de-efferented gastrocnemius of the rat. Pflugers Arch. 1973; 341:155-170. [PubMed: 4269823]

62. Albert F, Bergenheim M, Ribot-Ciscar E, Roll J-P. The Ia afferent feedback of a given movement evokes the illusion of the same movement when returned to the subject via muscle tendon vibration. Exp Brain Res. 2006; 172:163-174. [PubMed: 16421730]

63. Restuccia D. Somatosensory evoked potentials after multisegmental lower limb stimulation in focal lesions of the lumbosacral spinal cord. Journal of Neurology, Neurosurgery \& Psychiatry. 2000; 69:91-95. 
64. Vallbo AB, al Falahe NA. Human muscle spindle response in a motor learning task. J Physiol (Lond.). 1990; 421:553-568. [PubMed: 2140862]

65. Roll J-P, Albert F, Ribot-Ciscar E, Bergenheim M. 'Proprioceptive signature' of cursive writing in humans: a multi-population coding. Exp Brain Res. 2004; 157:359-368. [PubMed: 15007582]

66. Han J, Waddington G, Adams R, Anson J, Liu Y. Assessing proprioception: A critical review of methods. Journal of Sport and Health Science. 2016; 5:80-90. [PubMed: 30356896]

67. Ishikawa K, Ott K, Porter RW, Stuart D. Low frequency depression of the $\mathrm{H}$ wave in normal and spinal man. Exp Neurol. 1966; 15:140-156. [PubMed: 5934660]

68. Calancie B, et al. Evidence that alterations in presynaptic inhibition contribute to segmental hypoand hyperexcitability after spinal cord injury in man. Electroencephalography and Clinical Neurophysiology/Evoked Potentials Section. 1993; 89:177-186.

69. Schindler-Ivens S, Shields RK. Low frequency depression of H-reflexes in humans with acute and chronic spinal-cord injury. Exp Brain Res. 2000; 133:233-241. [PubMed: 10968224]

70. Vallery H, et al. Multidirectional transparent support for overground gait training. IEEE Int Conf Rehabil Robot. 2013; 2013:6650512-7. [PubMed: 24187327] 


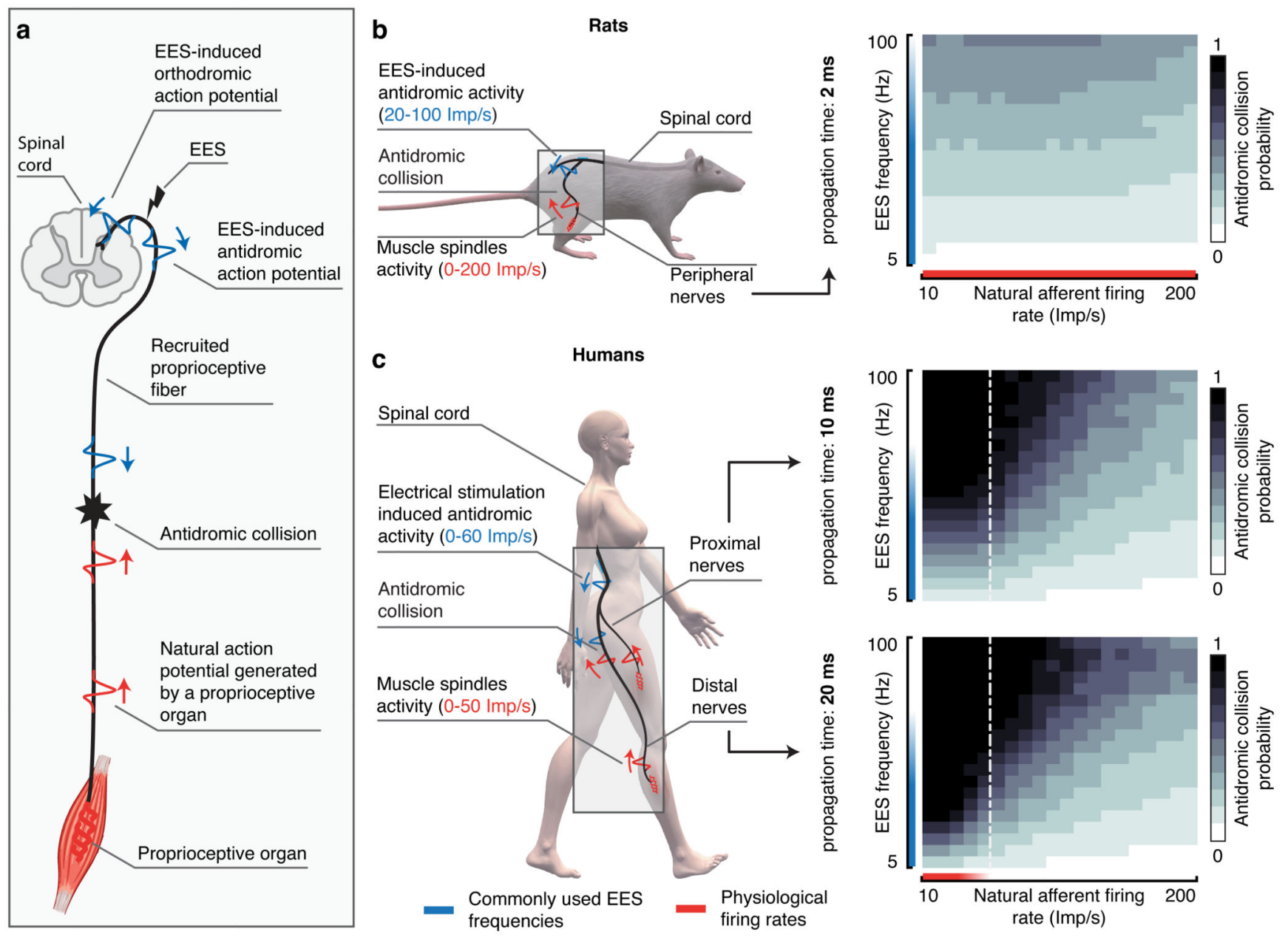

Figure 1. Probability of antidromic collisions during EES in rats and humans.

a, Schematic illustration of antidromic collisions between EES-induced antidromic action potentials and natural action potentials traveling along the recruited proprioceptive afferent fibers. b, c, Probability for a natural action potential to collide with EES-induced antidromic action potential in the proprioceptive afferent fibers of rats (b; action potential propagation time along the entire length of the fiber: $2 \mathrm{~ms}$ ) and in the proximal and distal proprioceptive afferent fibers of humans (c; action potential propagation time along the entire length of the fiber: 10 and $20 \mathrm{~ms}$, respectively). The probability is calculated as a function of EES frequency and natural firing rate along afferent fibers. EES frequencies that are commonly used to facilitate locomotion in rats and humans are highlighted in blue. Physiological proprioceptive firing rates reported in rats and humans are highlighted in red. The vertical dashed white line highlights the estimated maximum firing rate of human proprioceptive afferents during gait. Imp, impulse. 


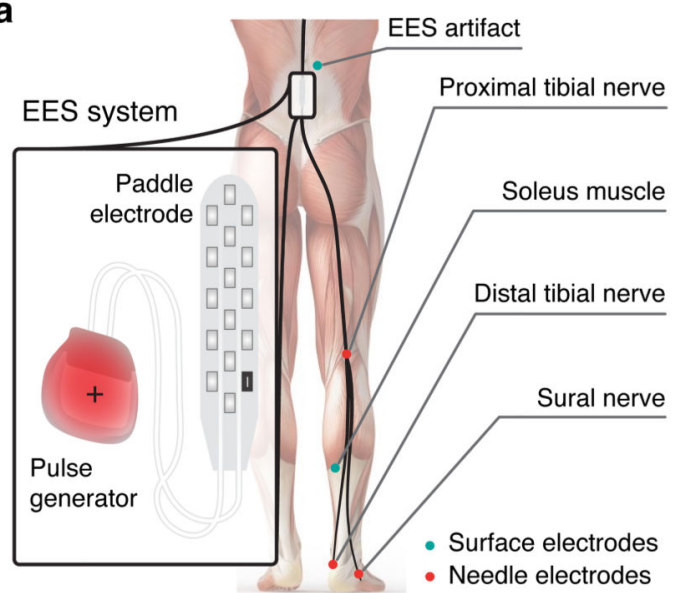

Subject \#2

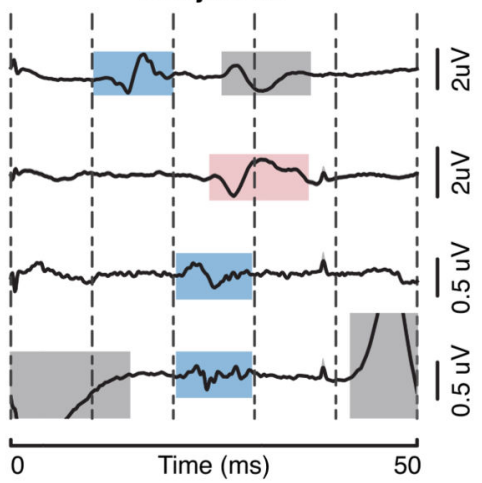

Subject \#3

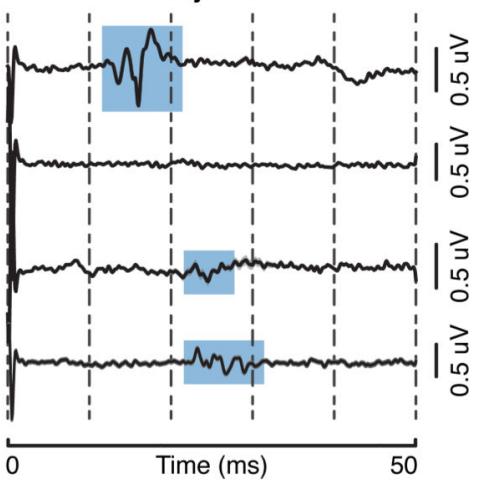

Sensory antidromic activity Motor orthodromic activity $\quad$ Far-field potential

b

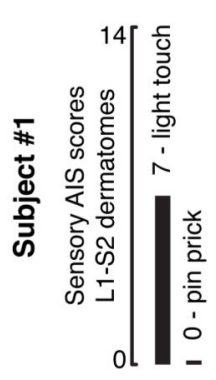

C

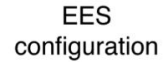

Experimental setup

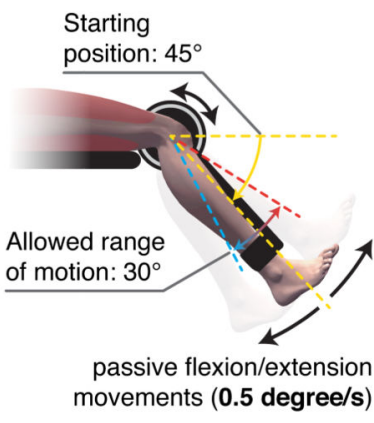

Experimental setup

EES
configuration

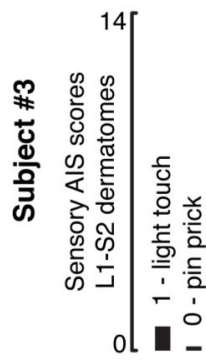

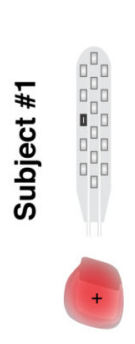

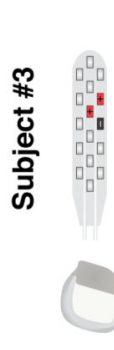

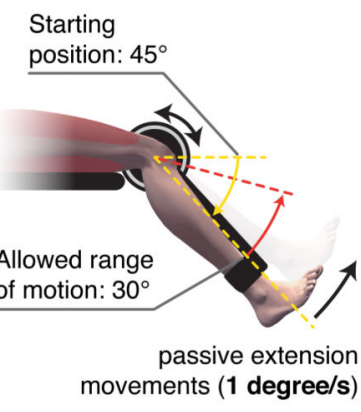
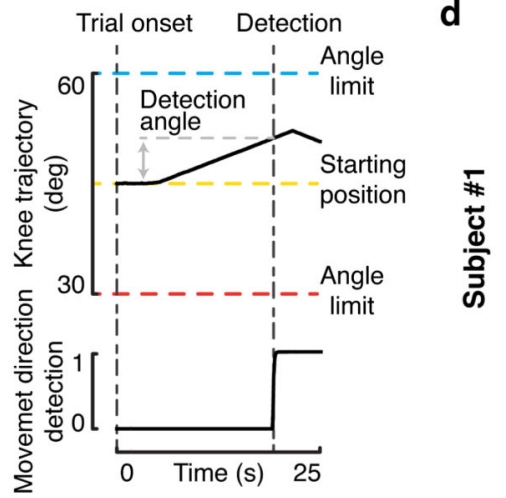
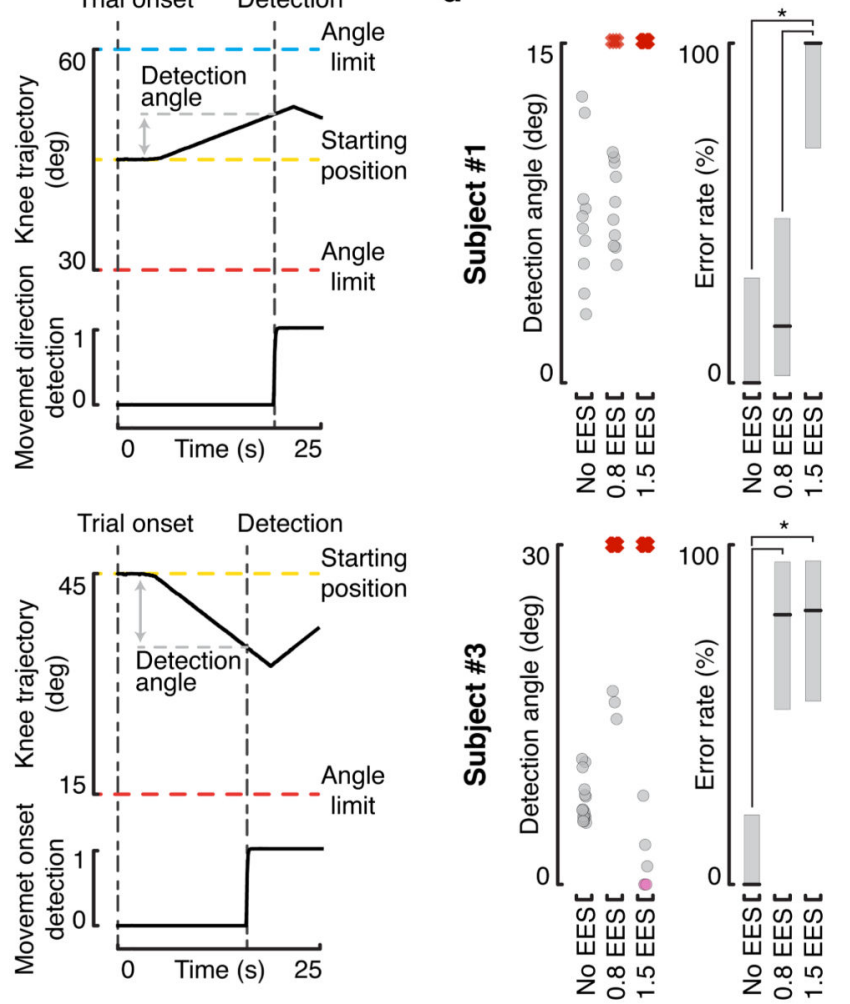

Figure 2. EES induces antidromic activity along proprioceptive afferents and disrupts proprioception.

a, Recordings of antidromic activity from sensory nerves during EES. Needle electrodes were inserted subcutaneously close to peripheral nerves and surface electrodes over the soleus muscle, as depicted in the scheme. Continuous EES $(20 \mathrm{~Hz}$, monopolar stimulation, black cathode and red anode) was delivered for approximatively one minute. Averaged evoked potentials $( \pm$ SEM, $n=1198$ and $n=1180$ independent measurements for subject \#2 and \#3, respectively). Evoked potentials highlighted in blue, red and grey were respectively classified as antidromic afferent volleys, efferent orthodromic activity, and far-field potentials (e.g. electromyographic activity of nearby muscles). b, Sensory subscores of the 
L1-S2 dermatomes for the two subjects that performed the threshold to detection of passive movement (TTDPM) test. c, Setup of the TTDPM test. Randomly selected flexion or extension movements were imposed to the knee joint of subject \#1 (top). A movement speed of 0.5 degree per second and a maximum allowed range of motion of 15 degrees was used. Subject \#3 (bottom) was not able to perceive movement direction. Hence, only the ability to detect extension movements was assessed. A movement speed of 1 degree per second and a maximum allowed range of motion of 30 degrees was used. EES configurations used to target knee flexor and extensor muscles were applied as indicated. d, Scatter plots reporting the detection angle and plots reporting the error rate (percentage correct trials $\pm 95 \% \mathrm{CI}, \mathrm{n}=$ 32 and $n=47$ independent measurements for subject \#1 and \#3, respectively) on the TTDPM test performance without EES and when delivering continuous EES $(50 \mathrm{~Hz})$ at 0.8 and 1.5 times muscle response threshold amplitudes. Grey dots report the detection angle for successful trials, while pink dots and red crosses indicate false positive and failure to detect movement within the allowed range of motion, respectively. $*, P<0.05$, Clopper-Pearson non-overlapping intervals, two-sided. 

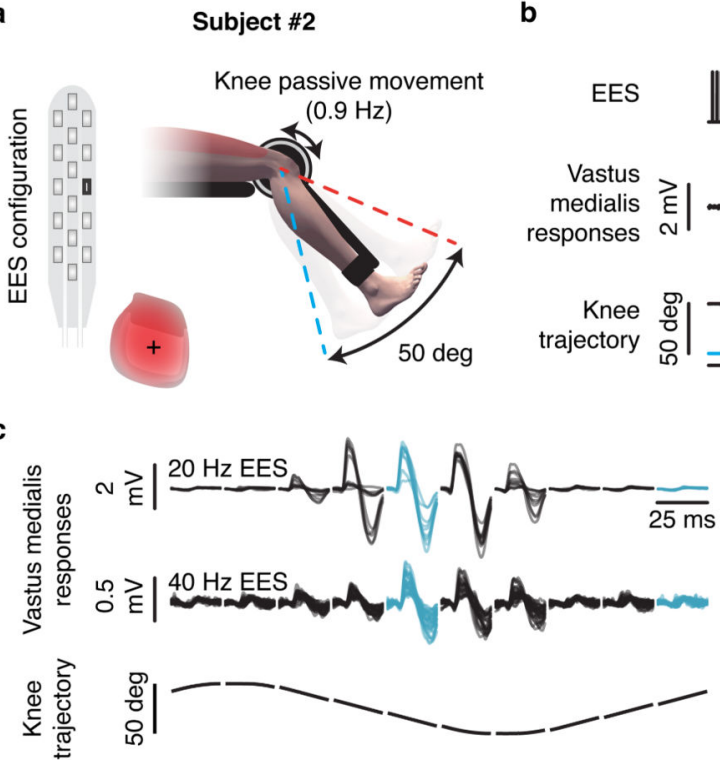

e

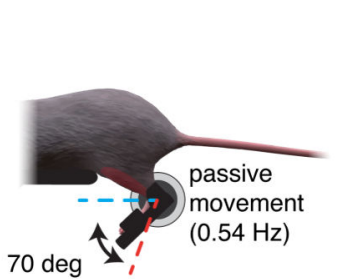

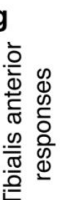
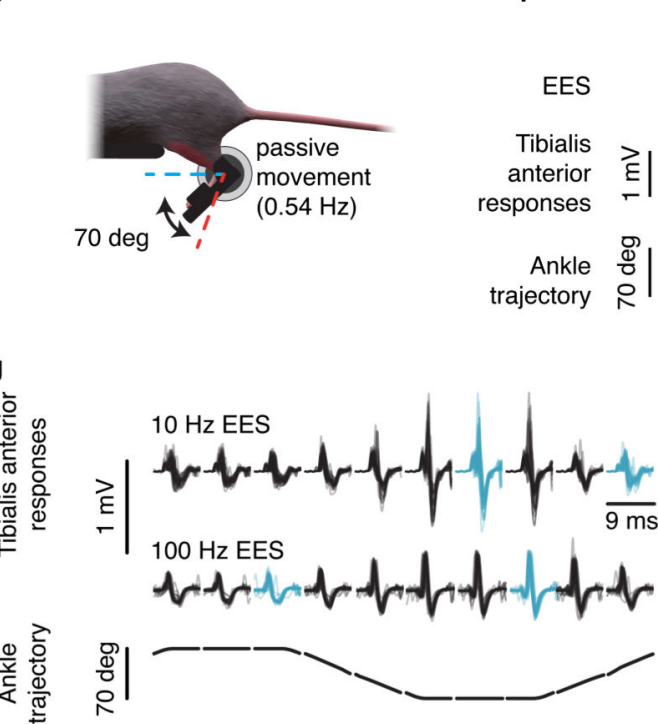

b

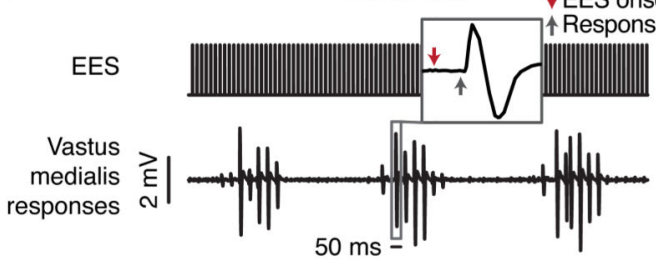

$\begin{aligned} \text { Knee } & \text { ס्ञ } \\ \text { trajectory } & 0\end{aligned}$

d

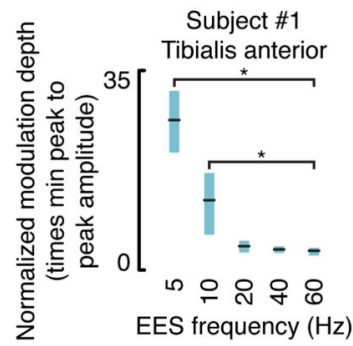

f

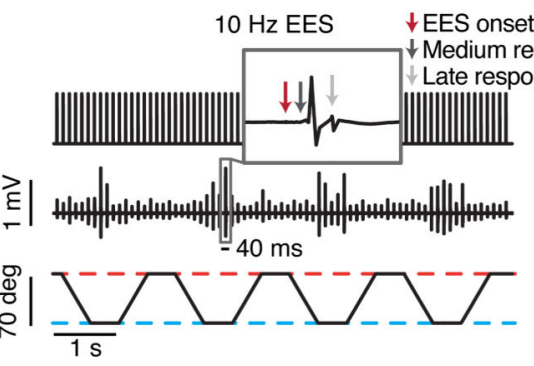

$\mathbf{h}$

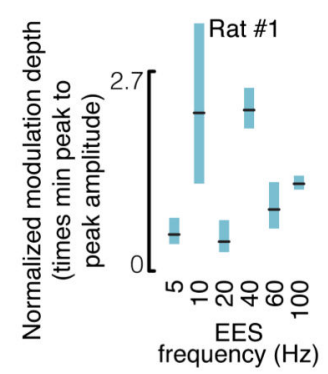

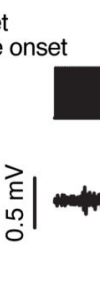

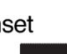
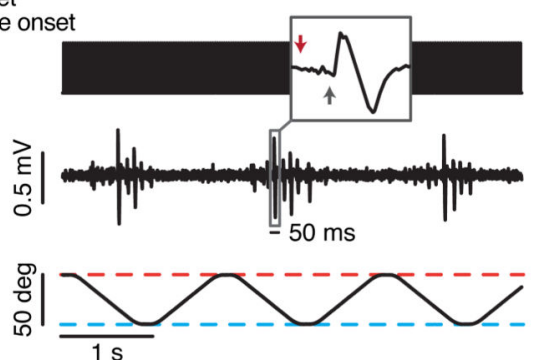

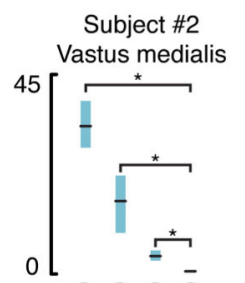

오 응우웅 EES frequency $(\mathrm{Hz})$

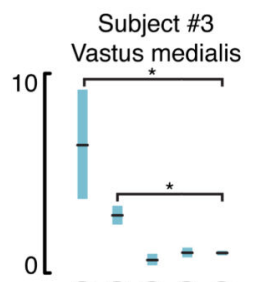

으 유 으 아 웅 EES frequency $(\mathrm{Hz})$

Figure 3. Effect of EES on the natural modulation of proprioceptive circuits during passive movements.

a, Configuration of the experimental setup for subject \#2. The subjects were secured in a robotic system that moved the ankle or knee joint passively within the reported range of motion. EES electrodes were configured to target a muscle that underwent stretching cycles during the selected joint movement, as highlighted in red. Configuration of the experimental setup for subjects \#1 and \#3 are reported in Supplementary Figure 2. b, Plots showing EES pulses, EMG activity of the vastus medialis, and changes in knee joint angle during passive oscillations of the knee for two different EES frequencies (20 and $40 \mathrm{~Hz}$ ) in subject \#2 similar results were obtained in subject \#1 and \#3. The same plots for $60 \mathrm{~Hz}$ are reported in Supplementary Figure 2. The rectangular windows highlight muscle responses induced by a single pulse of EES. Red and grey arrows depict the onset of the stimulation pulse and of the 
muscle response, respectively. c, The cycle of joint oscillation was divided into 10 bins of equal durations during which muscle responses were extracted and regrouped.

Superimposed muscle responses are displayed for each bin for two EES frequencies (subject \#2). Muscle responses used to compute the normalized modulation depth are depicted in light blue. d, Plots reporting the median and 95\% CI of the normalized modulation depth, for each EES condition tested and for the different subjects. The CI was bootstrapped (10000 iterations) over $\mathrm{n}=2344, \mathrm{n}=1080$, and $\mathrm{n}=2820$ muscle responses, respectively for subject \#1, \#2, and \#3. Low frequencies of stimulation often induced spasms in the muscles. Consequently, subjects \#2 and \#3 could not be tested with EES frequencies below 20 and 10 Hz, respectively. *, $P<0.05$, bootstrap, two-sided. e-h, Configuration of the experimental setup for rats with severe contusion SCI (250 kdyn) and results following the same conventions as in (b-d) for human subjects. Results in $\mathbf{f}$ and $\mathbf{g}$ are for rat \#1, similar results were obtained for all rats. The CI in $\mathbf{h}$ was bootstrapped (10000 iterations) over $\mathrm{n}=1834, \mathrm{n}$ $=1982, \mathrm{n}=1984$, and $\mathrm{n}=1983$ muscle responses, respectively for rat \#1, \#2, \#3, and \#4. 

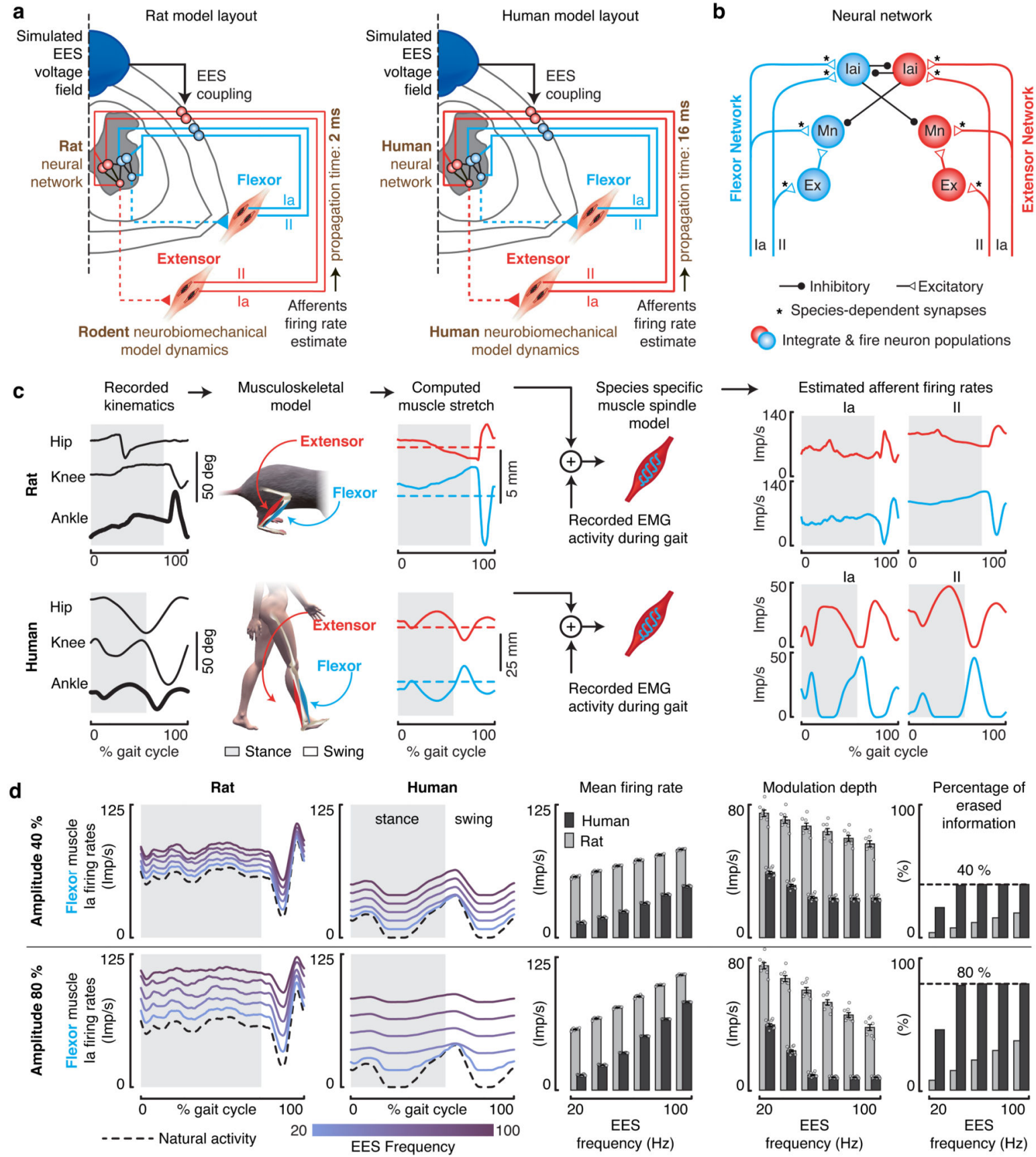

Figure 4. Impact of continuous EES on proprioceptive afferent firings during locomotion in rats and humans.

a, Layout of the computational models built for rats and humans. The components highlighted in brown are tuned to match the anatomical and physiological features of rats versus humans. b, Spiking neural network model of muscle spindle feedback circuits for a pair of antagonist muscles. Mn, motoneuron. Ex, excitatory interneurons. Iai, Ia-inhibitory interneurons. The synapses highlighted with an asterisk $\left(^{*}\right)$ are tuned to match the known properties of humans and rats. c, Estimated stretch profiles and afferent firing rates of ankle 
flexor and extensor muscles over an entire gait cycle in rats (top) and humans (bottom).

Similar results were obtained for $n=8$ gait cycles in rats, and $n=11$ gait cycles in humans.

d, Impact of EES on the predicted natural firing rate profiles of group-Ia afferents innervating a flexor muscle of the ankle during locomotion in rats (left) and humans (right). From left to right: averaged firing rate profiles of the simulated population of afferent fibers over one gait cycle, mean afferent firing rate ( \pm SEM, $n=8$ gait cycles in rats, $n=11$ gait cycles in humans), modulation depth of afferents firing rate profiles (mean \pm SEM, $n=8$ gait cycles in rats, $\mathrm{n}=11$ gait cycles in humans), and total amount of sensory information erased by EES. Results are reported over a range of EES frequencies. Top and bottom panels reports the results for EES amplitudes recruiting 40\% (top) or 80\% (bottom) of the entire population of modeled group-Ia afferents. 
a

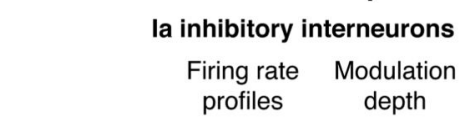

Rat computational model

motoneurons

firing rate mean firing

profiles rate when
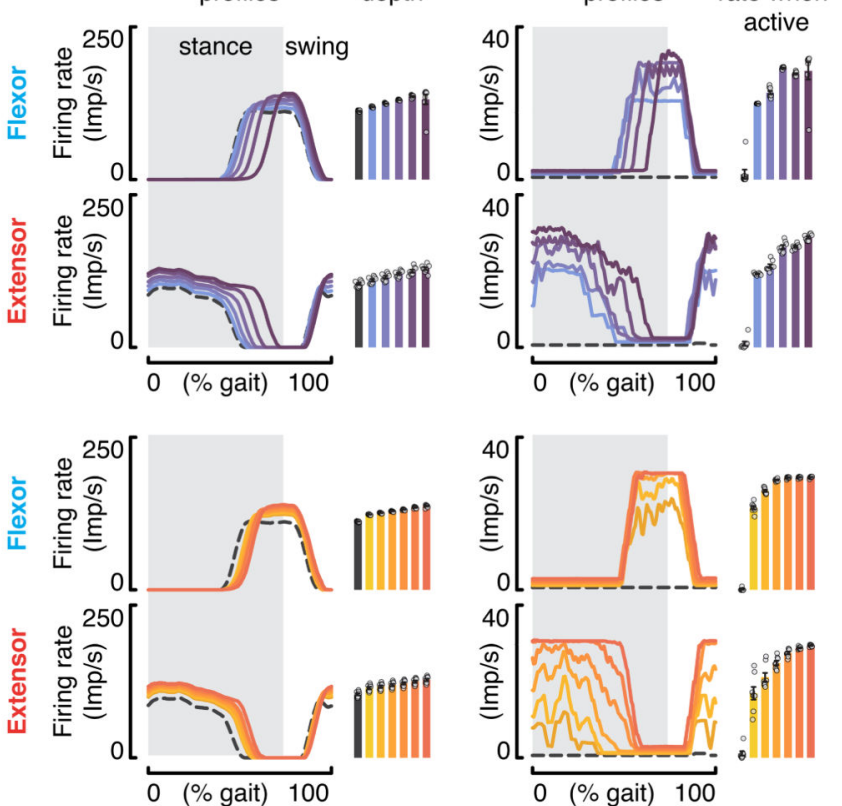

20

EES frequency $(\mathrm{Hz})$
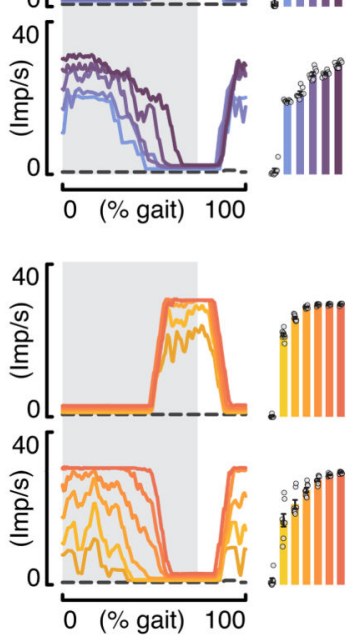

100 b
Human computational model

la inhibitory interneurons

firing rate modulation

profiles depth
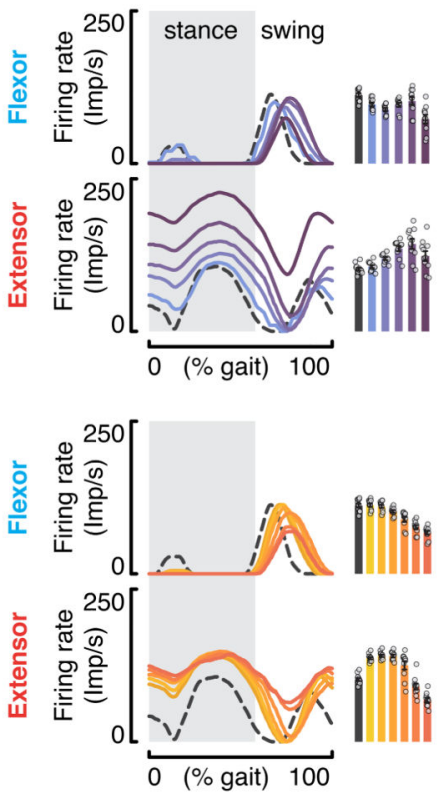

$40 \square 90$

EES amplitude (\% recruited la afferents) motoneurons

firing rate mean firing

profiles rate when
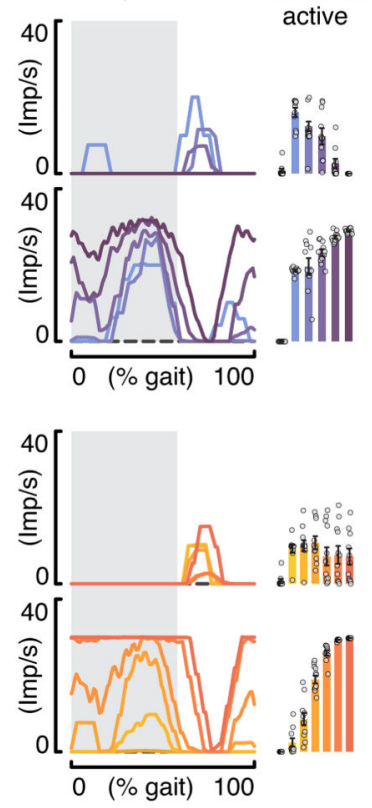

$\square$ Stance $\square$ Swing

Figure 5. Interactions between EES and muscle spindle feedback circuits during locomotion in rats and humans.

$\mathbf{a}, \mathbf{b}$, Impact of EES on the modeled natural activity of Ia-inhibitory interneurons and on the activation of motoneurons during locomotion in rats and humans. Left, average firing rate profiles and modulation depth of the Ia-inhibitory interneuron populations embedded in the flexor or extensor part of the neural network (mean \pm SEM., $n=8$ gait cycles in rats, $n=11$ gait cycles in humans). Right, average firing rate profiles and mean firing rate during the active phase for flexor and extensor motoneurons embedded in the flexor or the extensor neural network (mean \pm SEM., $n=8$ gait cycles in rats, $n=11$ gait cycles in humans). The impact of EES frequencies and amplitudes are reported in the top and bottom panels, respectively. EES amplitude was set to a value recruiting $65 \%$ of the modeled Ia afferents when EES frequency was scaled up, while EES frequency was set to $60 \mathrm{~Hz}$ when the amplitude was increased. 

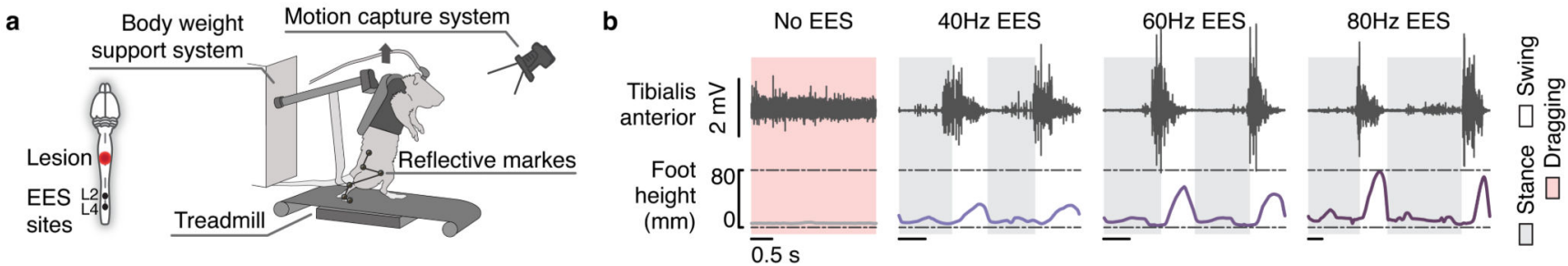

d

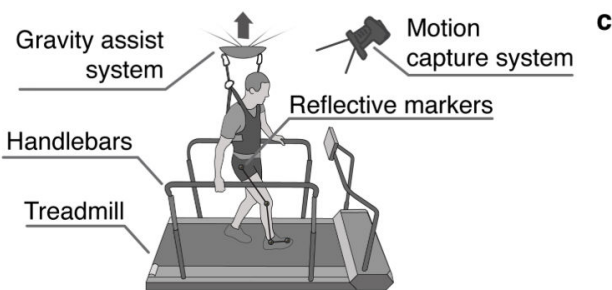

c
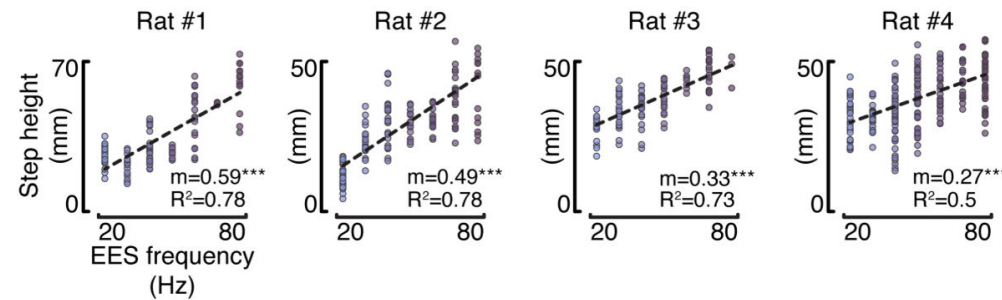

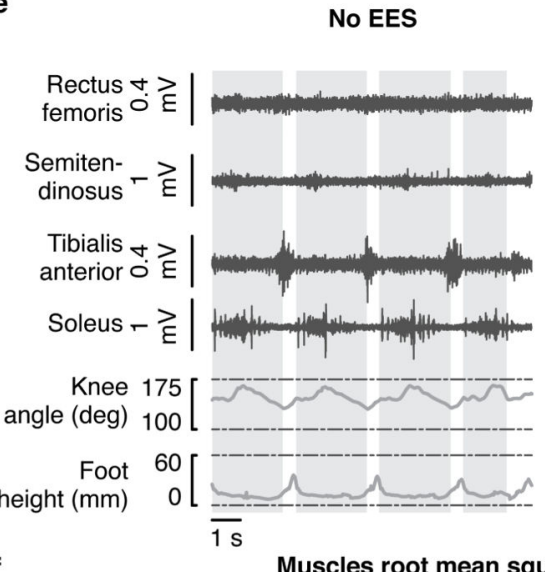

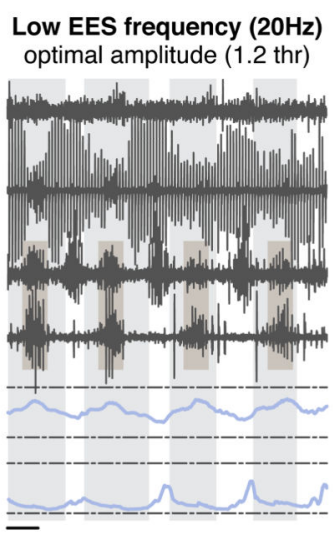

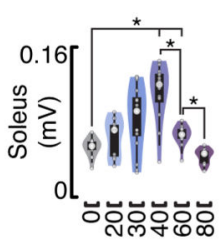

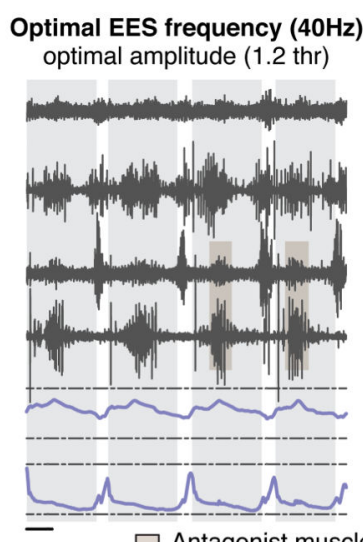

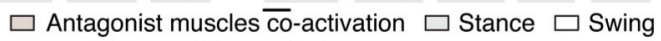
Kinematic features
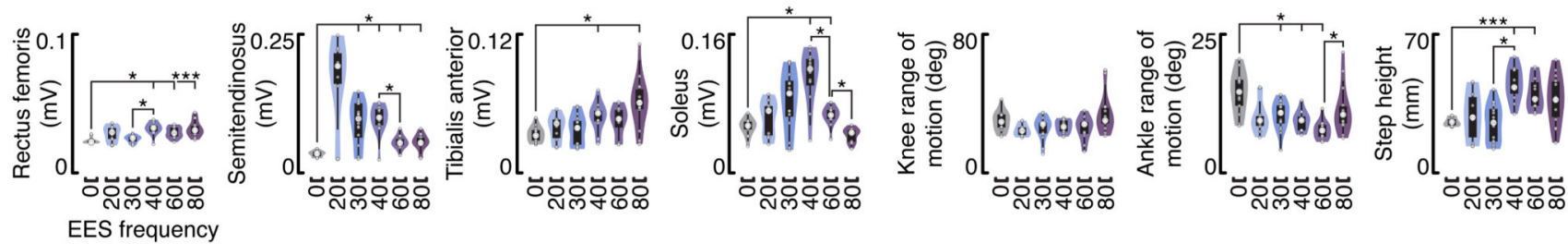

$(\mathrm{Hz})$

Figure 6. Impact of EES frequencies on muscle activity and leg kinematics during locomotion in rats and humans.

a, Experimental setup in rats. Rats with a severe contusion SCI were positioned in a robotic body weight support system located above a treadmill. Continuous EES was applied over L4 and L2 segments through chronically implanted electrodes secured over the midline of the dorsal spinal cord. b, EMG activity of the tibialis anterior muscle and foot height trajectory over two gait cycles without EES and with EES delivered at $40 \mathrm{~Hz}, 60 \mathrm{~Hz}$ and $80 \mathrm{~Hz}$ in rat $\# 1$ - similar results were obtained for rat \#2, \#3 and \#4. c, Scatter plots reporting the step height at different gait cycles for the tested EES frequencies $(n=111, n=139, n=101$, and $\mathrm{n}=231$ gait cycles, respectively for rat \#1, \#2, \#3, and \#4). Dashed lines report the linear regression between the EES frequency and the step height. Slope (m) and $\mathrm{R}^{2}$ are reported. $* * *, P<0.001$ two-sided Wald test slope $\neq 0$. d, Experimental setup in humans. Subjects were positioned in a gravity-assist system that provided personalized forward and upward 
forces to the trunk. Subjects were asked to step on the treadmill while holding the handlebars, since they were not able to step independently with the hands free. e, EMG activity of flexor (semitendinosus/tibialis anterior) and extensor (rectus femoris/soleus) muscles spanning the right knee and ankle joints, together with the changes in the knee ankle angles and foot elevation over four gait cycles without EES and with EES delivered at $20 \mathrm{~Hz}, 40 \mathrm{~Hz}$ and $80 \mathrm{~Hz}$ in subject \#1 - similar results were obtained for 49 gait cycles (analyzed in f). EES amplitude was set to 1.2 times the muscle response threshold. Notice the opposite modulation of EMG activity in extensor and flexor muscles with increase in frequencies together with co-activation of flexor with extensor muscles. $\mathbf{f}$, Violin plots reporting the root mean square activity of the recorded muscles, the range of motion of the knee and ankle angles, and the step height at different gait cycles for subject \#1 ( $\mathrm{n}=77$ gait cycles). Small grey dots represent the different data points, while the large white dots represent the median of the different distributions. Box and whiskers report the interquartile range and the adjacent values, respectively. $*, P<0.05$, ***, $P<0.001$, Wilcoxon rank-sum two-sided test with Bonferroni correction for multiple comparisons. The same results are reported for subjects \#2 and \#3 in Supplementary Figures 4 and 5. 


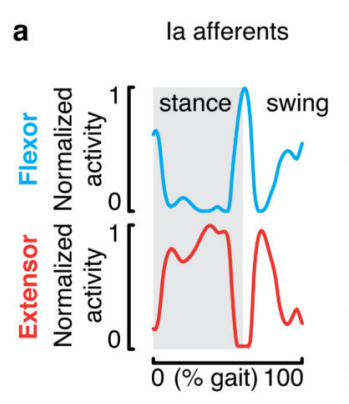

c
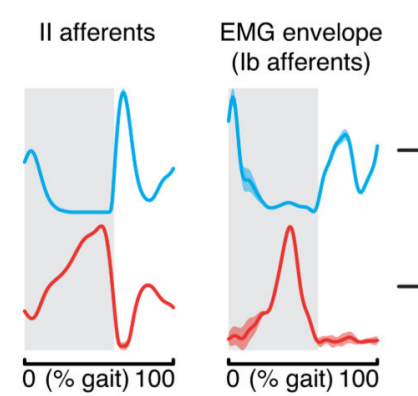

la afferent fibers
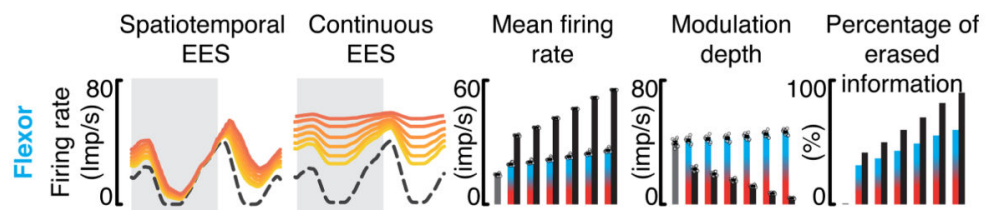

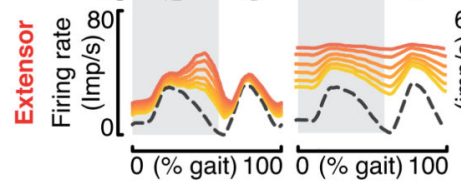

40

Stimulation amplitude

(\% recruited la afferents)

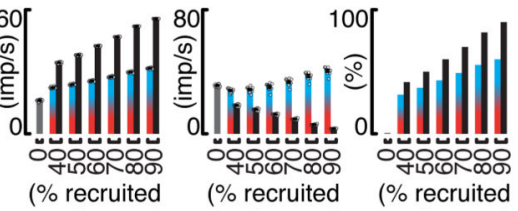

la afferents) la afferents) la afferents)
EES profile

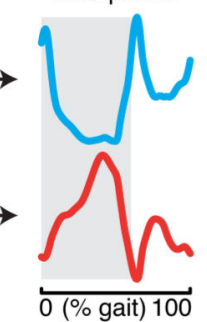

- Spatiotemporal EES b
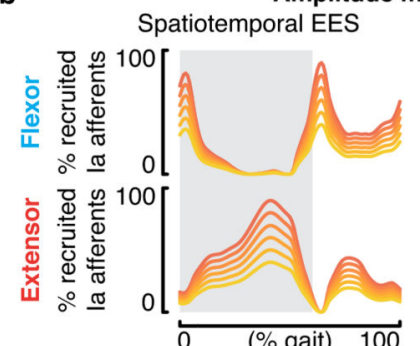

Continuous EES
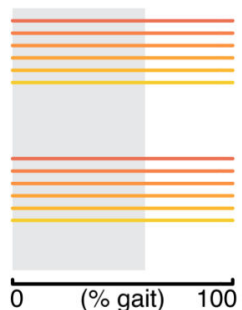

la inhibitory interneurons

Motoneurons

Firing rate Modulation profiles depth $\begin{array}{cc}\text { Firing rate } & \text { Mean firing } \\ \text { profiles } & \text { rate when }\end{array}$
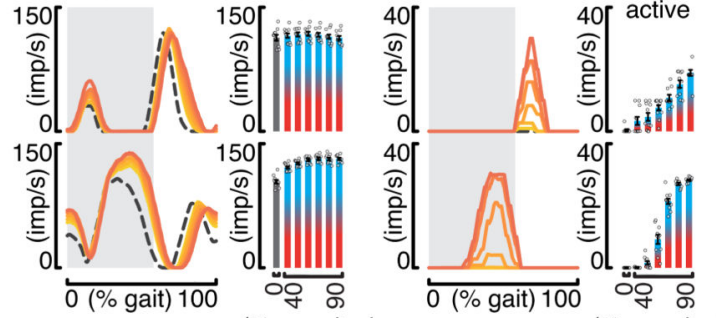
$50[$ onth है

。ㅇำ (\% recruited la afferents)

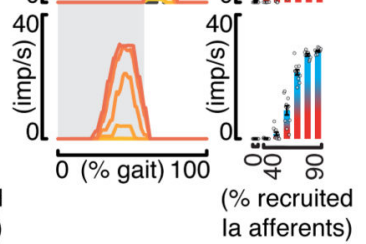

- Continuous EES $\square$ Stance $\square$ Swing

Figure 7. Spatiotemporal EES protocols encoding proprioceptive sensory information. a, Estimation of spatiotemporal stimulation profiles that match the natural flow of proprioceptive information generated from flexor and extensor muscles of the ankle during gait. From left to right: estimated averaged firing rate profiles of group-Ia, group-II and group-Ib (equivalent to the muscle activity) afferents over a gait cycle, and the sum of these profiles that yielded the estimated stimulation profiles. b. Percentage of primary afferents that are recruited when applying the estimated spatiotemporal stimulation profile and during continuous stimulation. c, Impact of the estimated spatiotemporal stimulation profile on the modulation of muscle spindle feedback circuits from flexor and extensor muscles, including from left to right: group-Ia afferents firings, bar plots reporting the averaged mean firing rate and modulation depth of primary afferents (mean \pm SEM., $n=11$ gait cycles), overall percentage of sensory information erased by EES, modulation of Ia-inhibitory interneurons, and motoneuron activity (mean \pm SEM., $\mathrm{n}=11$ gait cycles). For comparison, the impact of continuous EES on the group-Ia afferent firings is also reported. Results of simulations are shown for a range of EES amplitudes. Conventions are the same as in Figure 5. 
a

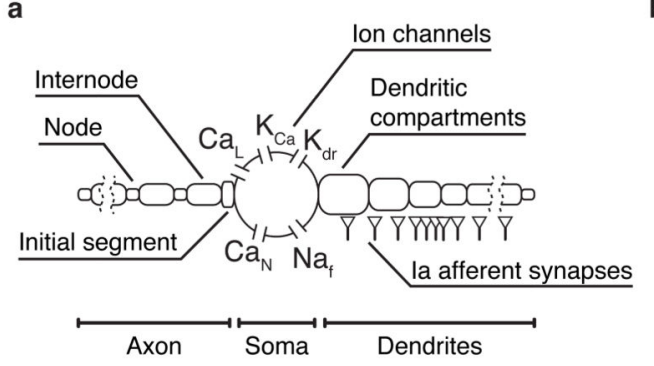

b

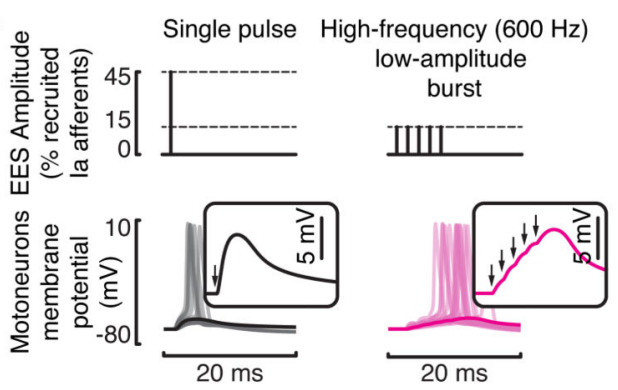

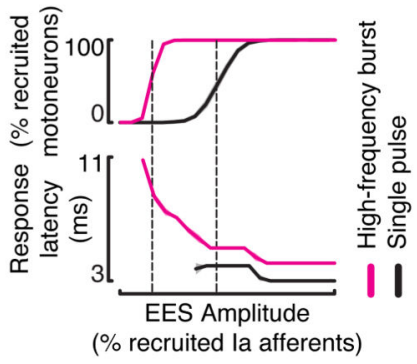

d

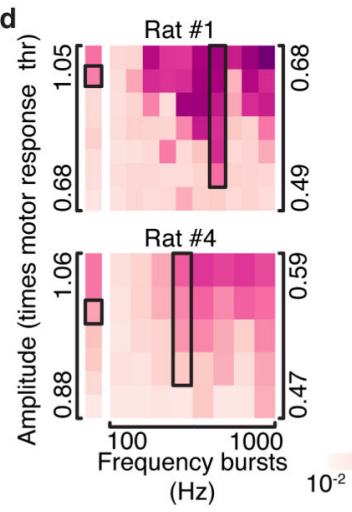

f

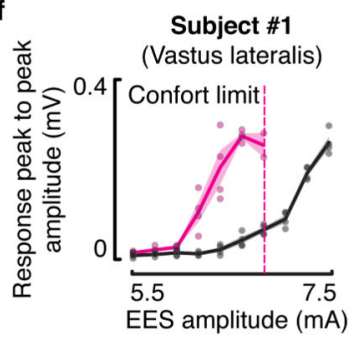

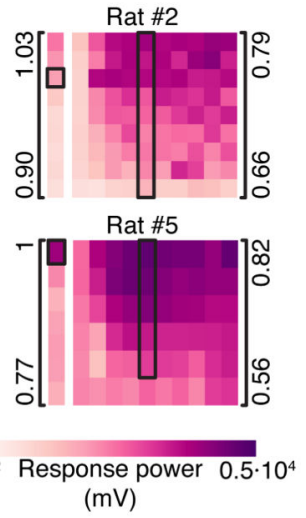

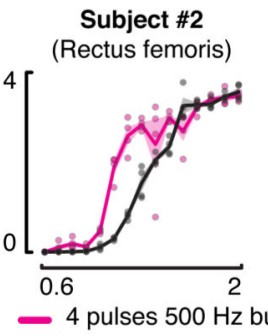

Rat \#3
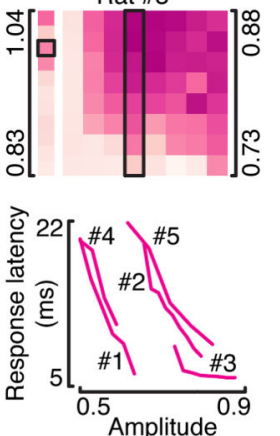

(times motor threshold)

Subject \#3

(Iliopsoas)

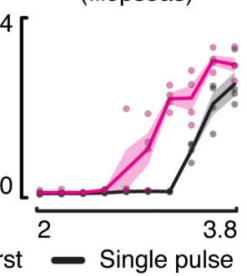

Figure 8. High-frequency low-amplitude bursts of EES recruit motoneurons through temporal summation of EPSPs.

a, Multicompartmental model of alpha motoneurons with realistic strength and distribution of group-Ia synaptic contacts. b, Simulations showing the response of motoneurons to a single pulse of EES at an amplitude recruiting $45 \%$ of the afferent population, and to highfrequency bursts $(5$ pulses, $600 \mathrm{~Hz}$ ) at an amplitude recruiting $15 \%$ of the afferent population. Windows show a zoomed view of the motoneuron membrane potential depolarizations in response to the pulses of EES (arrows). Right: plots showing the percentage of recruited motoneurons and the average (mean \pm SEM, $\mathrm{n}=5$ simulations with different random seed) latency before the onset of an action potential. c, Responses recorded from the tibialis anterior muscle following a single pulse of EES (left) and high-frequency bursts of EES (right) applied to the rat lumbar (L2) spinal cord with severe contusion SCI over a range of amplitudes and burst frequencies (rat \#1, shown for all rats in $\mathbf{d}$ ). The grey arrow indicates the responses induced by a single pulse of EES at the motor response threshold amplitude, emphasizing the need to deliver high amplitudes to elicit responses with single pulses compared to high-frequency bursts. d, Heatmaps representing the average power of motor responses $(n=4)$ to single pulses (column on the left) and high-frequency bursts (matrix on the right) of EES over a range of EES amplitudes and bursts frequencies, 
for 5 rats. EES amplitude is reported as a multiple of motor response threshold, amplitude corresponding to the response highlighted by the black box. The highlighted column corresponds to the bursts with a frequency inducing the largest motor responses. Right, latencies of motor responses elicited by EES bursts with the frequency highlighted in the black boxes, at increasing amplitudes. e, Motor responses recorded from the vastus lateralis muscle induced by single pulses (bottom) and high-frequency bursts of EES for different stimulation amplitudes (subject \#1). Shaded curves represent single trials ( $\mathrm{n}=4$ for each amplitude tested), solid curves represent the average responses. Arrows indicate the onset of the stimulation. $\mathrm{f}$, Plots representing the response peak to peak amplitudes (mean $\pm \mathrm{SEM}, \mathrm{n}$ $=4$ for each amplitude tested) as a function of EES amplitude, for both single pulses (black) and high-frequency bursts (pink) and for the different subjects. In subject \#1, EES amplitudes higher than $7 \mathrm{~mA}$ elicited uncomfortably powerful contractions and were thus not tested. 